\title{
A Study of Gravitational Lens Chromaticity using Ground-based Narrow band Photometry
}

\author{
A. M. Mosquera ${ }^{1,2}$, J. A. Muñoz ${ }^{1}$, E. Mediavilla ${ }^{3,4}$, and C. S. Kochanek ${ }^{2,5}$ \\ ${ }^{1}$ Departamento de Astronomía y Astrofísica, Universidad de Valencia, E-46100 Burjassot, \\ Valencia, Spain \\ ${ }^{2}$ Department of Astronomy, The Ohio State University, 140 West 18th Avenue, Columbus, \\ OH 43210, USA \\ ${ }^{3}$ Instituto de Astrofísica de Canarias, E-38200 La Laguna, Santa Cruz de Tenerife, Spain \\ ${ }^{4}$ Departamento de Astrofísica, Universidad de La Laguna, E-38200 La Laguna, Santa Cruz \\ de Tenerife, Spain \\ ${ }^{5}$ Center for Cosmology and Astroparticle Physics, The Ohio State University, 191 West \\ Woodruff Avenue, Columbus, OH 43210, USA
}

\begin{abstract}
We present observations of wavelength-dependent flux ratios for four gravitational lens systems (SDSS J1650+4251, HE 0435-1223, FBQ 0951+2635, and Q 0142-100) obtained with the Nordic Optical Telescope. The use of narrowband photometry, as well as the excellent seeing conditions during the observations, allows us to study their chromatic behavior. For SDSS J1650+4251, we determine the extinction curve of the dust in the $z_{L}=0.58$ lens galaxy and find that the $2175 \AA$ feature is absent. In the case of HE 0435-1223, we clearly detect chromatic microlensing. This allows us to estimate the wavelength-dependent size of the accretion disk. We find an R-band disk size of $r_{s}^{R}=13 \pm 5$ light days for a linear prior on $r_{s}^{R}$ and $r_{s}^{R}=7 \pm 6$ light days for a logarithmic prior. For a power-law size-wavelength scaling of $r_{s} \propto \lambda^{p}$, we were able to constrain the value of the exponent to $p=1.3 \pm 0.3$ for both $r_{s}^{R}$ priors, which is in agreement with the temperature profiles of simple thin disk models $(p=4 / 3)$.
\end{abstract}

Subject headings: accretion, accretion disks — dust, extinction — gravitational lensing: micro - quasars: individual (SDSS J1650+4251, HE 0435-1223, FBQ $0951+2635$, and Q 0142-100) 


\section{Introduction}

Gravitational lenses can act in many cases as cosmic telescopes. Since they can magnify the flux of background sources, they allow us to look far away in cosmic time, and to study in more detail the physics of the universe at cosmological distances, and beyond the resolution capabilities and detection thresholds of current telescopes. In particular, in gravitationally lensed quasars where variations in color are observed between their images, the "chromaticity" can be used to probe active galactic nucleus (AGN) and galactic structure using microlensing (see the review by Wambsganss 2006) and to study the properties of dust in intermediate- and high-redshift galaxies, as these are the effects leading to color differences between lensed images (see the review by Kochanek 2006).

The extinction effects are due to differences in the amounts and properties of dust in the lens galaxy near each image (Nadeau et al. 1991). In many cases the extinction curve of the dust in the lens galaxy can be accurately determined by measuring the wavelength dependence of the image brightnesses. This method is similar to the "pair method" (Massa et al. 1983), comparing the spectral energy distributions of stars with the same spectral type but different reddenings, used to study dust properties in the Milky Way (MW) and in nearby galaxies where the stars can be individually resolved. In the MW, most of the observed extinction curves are well fit by the so-called Cardelli et al. (1989, hereafter CCM) parameterization, a relationship that only depends upon the ratio of the total to the selective extinction, $R_{V}$. This parameter can take values from 2.1 to 5.8 depending on the line of sight, with a typical value of $R_{V} \sim 3.1$ (Draine 2003). The CCM parameterization includes a spectral feature at $2175 \AA$, but with some important differences that depend on environment. The Large Magellanic Cloud, for example, shows a weaker $2175 \AA$ feature in some regions (e.g., Nandy et al. 1981; Misselt et al. 1999; Gordon et al. 2003), while it is entirely absent for dust in the bar of the Small Magellanic Cloud (SMC, e.g., Prévot et al. 1984; Gordon et al. 2003). The (classical) "pair method" has been applied successfully up to distances $\sim 10$ Mpc (e.g., Bianchi et al. 1996), but cannot be used at longer distances because it requires observations of individual stars.

At cosmological distances, the pairs of lensed quasar images provide a powerful extension of the classical "pair method". Falco et al. (1999) studied extinction properties for a sample of 23 intermediate-redshift galaxies. They were able to estimate 37 differential extinctions, and also some absolute extinctions when the total absorption was large enough. The first measurement of an extinction curve at high redshift of comparable quality to those obtained in the MW was obtained by Motta et al. (2002). Muñoz et al. (2004) estimated the extinction laws in two intermediate-redshift galaxies and found unusual extinction curves compared with the MW. Mediavilla et al. (2005) extended the study of dust extinction 
properties to the far-UV, where extinction is strongest and different behaviors have been observed in different environments (Gordon et al. 2003). Elíasdóttir et al. (2006) used theoretical analysis and simulations to study the effects of extinction in 10 intermediateredshift galaxies and found no evolution of the dust properties with redshift even though, as they point out, a larger sample of lenses would be needed to reach a robust conclusion. Dai \& Kochanek (2009) measured the dust-to-gas ratio in six distant lens galaxies using Chandra observations to measure the difference in the gas column for comparison to the extinction differences, and they found a value consistent with the average ratio in the MW.

There are few other methods for studying dust extinction outside the MW and nearby galaxies. Another "non-classical" pair method compares reddened and unreddened photometrically similar Type Ia supernovae(SNe Ia; Riess et al. 1996; Perlmutter et al. 1997). Quasars with foreground absorbers have been used to measure the excess reddening produced by these systems. For instance, Murphy \& Liske (2004) studied the properties of dust associated with damped Ly $\alpha$ systems. Galactic environments were also traced with absorbers such as CIV (Richards et al. 2001), CaII (e.g., Wild et al. 2006), and MgII (e.g., Ménard et al. 2008). Finally, the spectra of the afterglows of gamma-ray bursts (GRBs) have also been used to determine extinction curves of dust in the host galaxies (e.g., Jakobsson et al. 2004). These methods have found that extinction curves similar to those in our neighbor galaxies are also found at high redshift. The $2175 \AA$ feature has been confirmed in several systems (Motta et al. 2002; Junkkarinen et al. 2004; Wang et al. 2004; Ellison et al. 2006), and its highest redshift $(z=2.45)$ detection to date was made by Elíasdóttir et al. (2009) using GRBs. The SMC extinction law also appears to reproduce the extinction in some intermediate- and high-redshift systems, such as the line of sights of many quasars (e.g., Hopkins et al. 2004), and to other GRB afterglows (e.g., Kann et al. 2006), although the sample of robustly confirmed SMC-like extinction laws is very small.

Besides extinction, microlensing-induced variability by stars and compact objects in the lens galaxy (see the review by Wambsganss 2006 and references therein) can also produce chromatic variations between the quasar images. The magnification produced by microlensing depends strongly upon the source size, with smaller emission regions showing larger variability amplitudes. In particular, since quasar accretion disk models (Blaes 2004, and references therein) all assume that the thermal emitting region is larger at longer wavelengths, we should observe different microlensing magnifications at different wavelengths (i.e., chromatic microlensing). Although non-negligible, this chromatic variation is more difficult to observe compared with achromatic microlensing, because microlensing magnification gradients on the scale of the source size are required to produce the effect (Wambsganss \& Paczynski 1991). However microlensing-induced chromaticity has been detected and analyzed in many lens systems (Anguita et al. 2008; Bate et al. 2008; Eigenbrod et al. 2008; 
Poindexter et al. 2008; Floyd et al. 2009; Mosquera et al. 2009; Blackburne et al. 2011). The sizes of the non-thermal emission regions have also been studied using the differences between optical and X-ray flux ratios (Pooley et al. 2007; Morgan et al. 2008; Chartas et al. 2009; Dai et al. 2009; Blackburne et al. 2011). These results showed that microlensing is a unique tool to zoom in on AGN and measure their physical properties. Unfortunately it is not always easy to separate extinction from chromatic microlensing when variations in color are observed.

One successful approach to studying chromatic microlensing is the use of narrowband photometry (Mosquera et al. 2009). Since the broad line emitting regions of quasars are generally too large to be microlensed (e.g., Abajas et al. 2002; Bentz et al. 2009), offsets should be observed between the flux ratios of adjacent continuum and emission line wavelengths when microlensing is present. These offsets cannot be accurately measured using broadband photometry, since each filter typically contains contributions from both emission regions. Narrowband filters can separate the two, and thus distinguish microlensing from extinction.

In this work, we examine the wavelength-dependent flux ratios of SDSS J1650+4251, HE 0435-1223, FBQ 0951+2635, and Q 0142-100 measured at eight different wavelengths and at several epochs using the Nordic Optical Telescope (NOT). These systems were selected because they have previously shown evidence for extinction or achromatic microlensing. The details of the observations and data reduction techniques are described in Section 2. The modeling and analysis of each individual system appears in Section 3. The conclusions and the main results are summarized in Section 4.

\section{Observations and analysis}

We observed the gravitational lens systems SDSS J1650+4251, HE 0435-1223, FBQ 0951+2635, and Q 0142-100 with the 2.56 m NOT located at Roque de Los Muchachos, La Palma, Spain. The images were taken with the $2048 \times 2048$ ALSFOC detector, which has a spatial scale of $0.188 \mathrm{arcsec} /$ pixel. We used seven narrowband filters, plus Bessel-I, which cover the wavelength interval 3510-8130 A. In the case of SDSS J1650+4251, a second round of monitoring observations was performed using the Liverpool Telescope (LT) with the $2048 \times 2048$ RATCam CCD, the scale of which is $0.135 \mathrm{arcsec} / \mathrm{pixel}$. We used the Sloan Digital Sky Survey (SDSS) ugriz filters covering the wavelength range from $3000 \AA$ to $10000 \AA$. Tables 1 and 2 provide a log of our observations and describe the filters. The data were reduced using standard IRAF procedures. After overscan and bias correction, a master flat-field image was created for each filter and then applied. Finally, frames of the same object were aligned and 
combined for each filter. Cosmic rays were removed when combining the frames by using a $3 \sigma$ crreject rejection algorithm.

We used imfitfits (McLeod et al. 1998; Lehár et al. 2000) to model the images and derive the magnitude differences between the quasar images as a function of wavelength. The quasar images were fit as point sources and the galaxy luminosity profile was described either with a de Vaucouleurs model or an exponential disk. The model consisted of the positions and intensities of all the components, as well as the parameters describing the lens galaxy morphology (effective/scale radius, ellipticity, and position angle). As these parameters were allowed to vary, the models were convolved with several point-spread functions (PSFs; typically three or four) defined by stars near each lens system and optimized by computing the $\chi^{2}$ to fit the observed image. If Hubble Space Telescope (HST) data were available, the component positions and the structure of the lens galaxy were fixed to the values derived from the HST data.

Since the main contribution to the residuals is caused by systematic errors arising from the PSF models rather than statistical fluctuations, we estimated the final magnitude differences from the model whose PSF best fits the combined frame. The final uncertainties in the magnitude differences were calculated by adding in quadrature two contributions: one that comes from the dispersion of the results from the PSFs and the other from the dispersion of the fits to the individual frames. In all cases, the residuals were negligible compared to the image fluxes. Tables 3 and 4 report the results, and the details of the individual models are discussed in the next section.

\section{The Individual Systems}

\subsection{SDSS J1650+4251}

SDSS J1650+4251 is a two image lens discovered by Morgan et al. (2003). It has a source redshift of $z_{s}=1.547$. The separation of the two QSO components is 1 .'2 with B-band magnitudes of 17.8 and 20.0. The detection of MgII and FeII absorption lines in the spectra of the lensed quasar suggests a lens redshift of $z_{L}=0.58$ (Morgan et al. 2003), although a resolved spectrum of the lensing galaxy is needed to confirm this value. The time delay for the system is estimated to be $\sim 1$ month (Morgan et al. 2003; Vuissoz et al. 2007).

The first series of observations of SDSS J1650+4251 were obtained with the NOT telescope using eight mostly narrowband filters. Due to the good seeing conditions, the two quasar components A and B (the brightest and the faintest, respectively) were clearly resolved. An unassociated galaxy 4 arcsec south of image A was also detected. To estimate the 
I-band magnitudes, we fit the two components as point sources and modeled the lens galaxy and the nearby "external" galaxy as either a de Vaucouleurs model or an exponential disk. Since the lens galaxy is very faint, it is difficult to distinguish between these two luminosity profiles. The best fit was obtained with a de Vaucouleurs profile for the lens galaxy and an exponential disk for the nearby galaxy. The excellent seeing conditions (0!6) allow us to determine the relative positions and brightnesses of the quasar images, as well as the position of the lens galaxy and its flux. The results for the relative component positions in Table 5 are in agreement with the ones obtained by Morgan et al. (2003). The broad I-band filter is the only one in which the lens galaxy is well detected, appearing as a clear residual if we use a model excluding it.

In the seven narrowband filters, the contribution from the lens galaxy is very small, and the lens system can be modeled simply as two point sources. The resulting fits are excellent, as illustrated by the residuals for the Strömgren-y image shown in Figure 1. The peak residuals for the images are less than $1.7 \%$ of the peak intensity of image A. The absence of the lens galaxy in the residuals, even at wavelengths beyond the $4000 \AA$ break, is consistent with the expected level of contamination. Assuming that the calculated flux ratios (with and without galaxy) are well determined in the I-band, we can estimate the contamination in the bluer filters using a quasar spectrum template for the images and an early-type spectrum template for the lens galaxy (Assef et al. 2010). We find that the lens galaxy contamination in the flux ratios would be at most $\sim 0.04$ mag in the bluer filters, within the level of the model uncertainties. The photometric results appear in Table 4 and are plotted as a function of wavelength in Fig. 2 (black squares). In Figure 2 we have also included the results of Morgan et al. (2003) (empty triangles), which are in good agreement with the ones we obtained.

To model the effects of differential extinction we fit the magnitude differences as a function of wavelength following Falco et al. (1999) as

$$
m_{B}(\lambda)-m_{A}(\lambda)=\Delta M+\Delta E R\left(\frac{\lambda}{1+z_{l}}\right),
$$

where $\Delta M=M_{B}-M_{A}$ is the relative magnification, $\Delta E(B-V)=E_{B}(B-V)-E_{A}(B-V)$ is the differential extinction, and $R(\lambda)$ is the mean extinction law. The fits can also be done as a function of the "dust redshift" (Jean \& Surdej 1998), either to estimate the lens redshift or as an added confirmation of extinction. All these quantities can be determined without needing to know the intrinsic spectrum of the quasar, $m_{0}(\lambda)$. We use a $\chi^{2}$ statistic for the fits and either the CCM (1989) parameterized models for the Galactic extinction curve or the Fitzpatrick \& Massa (1990) model with its parameters set to the values found by Gordon et al. (2003) for the average extinction in the SMC. The results are shown in Figure 2. 
Our best fit for the data with $z_{\text {dust }}=0.58$ is obtained for an SMC extinction law with $\chi_{d o f}^{2}=0.081$, where $\chi_{d o f}^{2}$ is $\chi^{2}$ per degree of freedom. The parameters for this fit are $\Delta M=1.8 \pm 0.1$ and $\Delta E=0.10 \pm 0.02$ at $1 \sigma$. For the CCM extinction model with $R_{V}=3.1$, we find $\chi_{d o f}^{2}=2.2$, which is a significantly worse fit because the Str-u point is in conflict with the presence of a $2175 \AA$ feature. Excluding the Str-u point, the CCM model would also fit well $\left(\chi_{d o f}^{2}=0.03\right)$.

The alternative to extinction as an explanation for the wavelength-dependent flux ratios is chromatic microlensing. We view this as a less likely explanation because the wavelength dependence observed in the NOT data is consistent with that observed $\sim 4$ months earlier by Morgan et al. (2003) and $\sim 3$ years later in our LT observations, as shown in Figure 3 , There is a wavelength-independent shift of $\sim 0.2 \mathrm{mag}$ that could be due to microlensing, but the chromatic structure is unchanged. Since the observations made at the LT cover a period larger than the expected time delay and no significant variations were observed between the different nights, the magnitude shifts are probably not due to intrinsic variability in the quasar modulated by the time delay.

\subsection{HE $0435-1223$}

HE 0435-1223 was discovered by Wisotzki et al. (2000) in the Hamburg/ESO survey for bright QSOs and later identified as a gravitational lens (Wisotzki et al. 2002). In this quadruple system, a background quasar with a redshift of $z_{S}=1.689$ is gravitationally lensed by an early-type galaxy at $z_{L}=0.4541$ (Morgan et al. 2005). Integral-field spectrophotometry (Wisotzki et al. 2003) showed no evidence for differential extinction, while there was evidence for microlensing. Kochanek et al. (2006) also observed microlensing variations

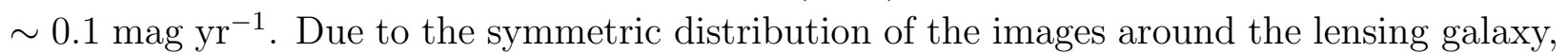
the time delays of $\Delta t_{A B}=-8.00_{-0.82}^{+0.73}$ days, $\Delta t_{A C}=-2.10_{-0.71}^{+0.78}$ days and $\Delta t_{A D}=-14.37_{-0.85}^{+0.75}$ days (Kochanek et al. 2006) are relatively small. Courbin et al. (2010) found similar time delays using a longer monitoring period but with larger formal uncertainties.

Observations of this system were made using the narrowband filter set at the NOT on two nights separated 15 days (see Table 1), and the photometric results for the two epochs agree at the 1- $\sigma$ level. Figure 4 shows the results of the PSF photometry for one of those nights, in which the lens galaxy was modeled with a de Vaucouleurs profile, and the quasar images were included as point sources. Their relative positions and the structure of the lens

\footnotetext{
${ }^{1}$ We neglect the somewhat offset I-band point. We think that it is discrepant because the lens galaxy was slightly oversubtracted.
} 
galaxy were fixed to the HST values (Kochanek et al. 2006).

The behavior of the flux differences in Figure 4 looks very similar to what we found when studying Q 2237+0305 (Mosquera et al. 2009), suggesting that we have detected chromatic microlensing in another quadruple system. Considering the quasar redshift, the Strömgren-u and Strömgren-v filters are the only ones affected by the broad emission lines of the quasar. The Ly $\alpha$ emission line contributes about $38 \%$ of the flux in the Strömgren-u filter, and part of the CIV emission line extends over the full width of the Strömgren-v filter based on the SDSS composite quasar spectrum (Vanden Berk et al. 2001). Therefore, in a system affected by microlensing, these two filters will be offset from the continuum flux ratios at those wavelengths, because the broad line regions are much larger than the length scales for microlensing (e.g., Abajas et al. 2002; Bentz et al. 2009).

Looking in Figure 4 at the filters that are not affected by emission lines, it appears that chromatic microlensing is affecting image A. The observed chromaticity between the bluest (Str-b) and the reddest (I-band) filters is $(\Delta m)_{I-b}=0.20 \pm 0.09$. As none of $\mathrm{B}$, C, or D shows significant signs of a wavelength dependence, even at wavelengths contaminated by emission lines, achromatic microlensing must be weak for these images. This is supported by the R-band light curves obtained with the SMARTS 1.3 telescope located at the Cerro Tololo Inter-American Observatory, in Chile (Blackburne \& Kochanek 2010). Figure 5 shows the brightness fluctuations observed in the difference $\left(m_{B}-m_{A}\right)$, once a time delay correction of -8.0 days was applied, and time-dependent changes are not seen in the other two differences $\left(m_{B}-m_{C} \sim 0.05\right.$ and $\left.m_{B}-m_{D} \sim-0.25\right)$. Thus, image A seems to have been undergoing a microlensing event near the time of our NOT observations, while no-microlensing variations were observed in B, C, and D. Our flux ratios for the redshift zero $\mathrm{H} \alpha$ filter agree well with the similar-wavelength SMARTS R-band observation (Figure 5, filled squares).

We modeled the microlensing of image $\mathrm{A}$ as follows. First, we fit a simple singular isothermal ellipsoid plus external shear model to the HST positions from Kochanek et al. (2006) using the lensmodel package (Keeton 2001) to determine a convergence and shear for image $\mathrm{A}$ of $\kappa=0.43$ and $\gamma=0.39$. Second, we generated microlensing magnification patterns using the inverse polygon mapping method of Mediavilla et al. (2006). We generated patterns with stellar mass fractions of $\kappa_{*} / \kappa=0.01,0.05,0.1,0.15,0.2,0.25$. We used stellar masses of $M=1 M_{\odot}$, an outer scale of $20 r_{E}$ and a dimension of $2048 \times 2048$ pixels to get a resolution of $0.01 r_{E}$ /pixel. With the present data we cannot determine $M$, and our size estimates can be rescaled as $\left(M / M_{\odot}\right)^{1 / 2}$. Finally, we estimated the R-band microlensing magnification of image A assuming that the H-band fluxes from Kochanek et al. (2006) represented the true flux ratios. The $\mathrm{H}$-band data are the reddest ones reported in the literature for HE $0435-1223$, and will be less affected by extinction and chromatic microlensing. Even 
though chromatic effects could be contaminating the H-band measurements, our observations support the hypothesis that they are negligible. The H-band magnitude differences, $\left(m_{B}-\right.$ $\left.m_{C}\right)_{H}=-0.02 \pm 0.04$ and $\left(m_{B}-m_{D}\right)_{H}=-0.23 \pm 0.06$, match the respective SMARTS R-band differences (see Figure 5). Since from the NOT chromatic data we know that neither $B, C$, nor $D$ is significantly microlensed, the H-band flux ratios should be a good estimate of the real fluxes of the images. This means that at R-band image $\mathrm{A}$ is magnified by microlensing by $\Delta M_{R}=-0.19 \pm 0.04$. The differential microlensing of image A between the Str-b and R bands was simply estimated from the observed chromatic microlensing, and is $\Delta M_{b-R}=-0.13 \pm 0.08$.

Finally, we convolved the magnification patterns with Gaussian source profiles $\left(\propto \exp \left(-r / 2 r_{s}\right)\right)$ of varying size $r_{s}^{R}$ to model the R-band and $r_{s}^{b}=r_{s}^{R}\left(\lambda_{b} / \lambda_{R}\right)^{p}$ for the Str-b band to compute the probability of reproducing $\Delta M_{R}$ and $\Delta M_{b-R}$ as a function of the size $r_{s}^{R}$, the powerlaw index $p$, and the stellar fraction $\kappa_{*} / \kappa$ (see Mosquera et al. (2009) for details on the probability distribution calculations). We adopted $p=\frac{i}{3}, i=1, \ldots, 6$, and $r_{s}^{R}=(1+2 i)$ light-days, $i=0, \ldots, 12$. Figure 6 (solid lines) shows the probability distribution $P\left(r_{s}^{R}, p\right)$ for the different values of the stellar fraction $\alpha=\kappa_{*} / \kappa$. The contours correspond to $15 \%, 47 \%$, $68 \%$, and $90 \%$ confidence intervals. The integrated values of the probabilities, $P_{\alpha}\left(r_{s}^{R}\right)$ and $P_{\alpha}(p)$, are shown in Figures 7 and 8 , respectively, for the considered stellar fractions. If we adopt the likelihood maximum as our estimator for $r_{s}^{R}$ and $p$, they can be constrained at $68 \%$ confidence for $\alpha<0.15$ (Table 6). For $\alpha>0.15$ the shape of the probability distributions does not allow us to determine the corresponding uncertainties. However, we know from Mediavilla et al. (2009) and from time delay measurements (Kochanek et al. 2006) that low values of $\kappa_{*} / \kappa \lesssim 0.2$ are favored. Therefore we calculated $P\left(r_{s}^{R}\right)$ and $P(p)$ using the probability distribution of the stellar mass fraction found by Mediavilla et al. (2009) as a prior (solid line in Figures 9 and 10), interpolating over the probability distributions since the sampling in $\alpha$ is not uniform. From these weighted probability distributions we estimate that the disk size and the power-law slope are $r_{s}^{R}=13 \pm 5$ light days and $p=1.3 \pm 0.3$ at $68 \%$ confidence. We also estimated these values for a logarithmic prior on $r_{s}^{R}$ (Table 6) just by dividing by $r_{s}^{R}$ the distributions obtained with a uniform prior. The probability distributions $P\left(r_{s}^{R}, p\right)$ using this prior, and the corresponding integrated values $P_{\alpha}\left(r_{s}^{R}\right)$ and $P_{\alpha}(p)$, are also shown in Figures 6, 7, and 8, respectively (dashed lines). In this case we found a disk size of $r_{s}^{R}=7 \pm 6$ light days and a similar slope $p$. Pooley et al. (2007, 2009) and Blackburne et al. (2011) found similar disk sizes to these estimates by comparing optical and X-ray flux ratios, and similar results were found by Morgan et al. (2008, 2010) modeling the R-band light curves (they estimated $r_{s}^{R}=15_{-9}^{+23}$ light days for a face-on quasar). The slope $p$ for the wavelength dependence is in agreement with the Shakura \& Sunyaev (1973) simple disk model $(p=4 / 3)$. Blackburne et al. (2011) also found solutions compatible with our results 
for HE $0435-1223$ with $p=0.55 \pm 0.49$ and $p=0.67 \pm 0.55$ for a linear and for a logarithmic prior, respectively, although they found a shallower average slope for their full sample of lenses.

If we compare the probability distribution $P(\alpha)$ without the Mediavilla et al. (2009) prior, it increases monotonically with $\alpha$ (Figure 11, dashed line). This is the expected behavior for $P(\alpha)$, since a single epoch chromatic microlensing detection likely introduces a strong bias toward large $\alpha$ values. With $\alpha$ large there are many more regions with the strong micro-magnification gradients needed to produce the observed chromaticity. This is the reason why we cannot constrain the stellar fraction with our procedure. When we include the Mediavilla et al. (2009) prior, the resulting $P(\alpha)$ resembles that prior (Figure 11, solid line). In the end, our procedure is essentially equivalent to computing the probability

distributions $P\left(r_{s}^{R}\right)$ and $P(p)$ for a fixed value of $\alpha \simeq 0.1$ that is also consistent with the time delays.

\subsection{FBQ $0951+2635$}

The gravitational lens system FBQ 0951+2635 was discovered by Schechter et al. (1998). It has two quasar images separated by $1^{\prime \prime} 1$, and the time delay between them is estimated to be approximately two weeks (e.g., Jakobsson et al. 2005). The quasar redshift is $z_{S}=1.246$. However the redshift of the lens galaxy was much more difficult to measure, since it was very difficult to disentangle its flux from the quasar images. Kochanek et al. (2000) suggested a value of $z_{L}=0.21$ from the position of the lens in the fundamental plane, and this was spectroscopically confirmed to be $z_{L}=0.260 \pm 0.002$ by Eigenbrod et al. (2007). Indications of microlensing in the system were found by several authors (e.g., Schechter et al. 1998; Jakobsson et al. 2005), but the chromatic behavior of FBQ $0951+2635$ is still not well understood. This system was observed during one night at the NOT (see Table 1) using our narrowband filter set. We fit the data using point sources for the quasar images, and the lens galaxy was included in the I-band model as a de Vaucouleurs profile. Figure 12 shows the result of our photometry compared with HST measurements at two different epochs (CASTLES and J. A. Muñoz et al. 2011, in preparation).

If we focus on the NOT data in Figure 12 (open squares), our results are compatible with no chromaticity. However, our data shed little light on the chromatic behavior of FBQ 0951+2635, because almost all the filters contain strong emission lines. The iac\#29 filter corresponds to the OIII line, iac\#28 is blended with MgII, the [NeIV], FeIII and [OII] lines lie in the Str-y filter, Str-v is affected at $\sim 50 \%$ by CIII], and the Str-u filter contains SiIV, OIV], CIV, and HeII. In any case, our results as well as those obtained by J. A. Muñoz 
et al. (2011, in preparation; Figure 12), confirm the lack of extinction because no chromatic fluctuations are observed. The J. A. Muñoz et al. (2011, in preparation) flux ratios are shifted by $\sim 0.1 \mathrm{mag}$, while the earlier CASTLES observations show a larger shift and a significant wavelength dependence, suggesting that there is significant microlensing in this system. In any case further observations are needed to better understand the chromatic behavior in this system.

\subsection{Q $0142-100$}

The gravitational lens Q 0142-100 (UM 673) was discovered by Surdej et al. (1987). It is a doubly imaged quasar with components separated by 2 .'2. The source redshift is $z_{S}=2.72$ (MacAlpine \& Feldman 1982), and absorption lines detected in the quasar spectra suggest a lensing galaxy at $z_{L}=0.49$ (e.g., Surdej et al. 1987). Several authors have discussed the chromatic behavior of this lens system (Falco et al. 1999; Wisotzki et al. 2004; Elíasdóttir et al. 2006), but its nature is still a matter of discussion. The photometric data obtained by Nakos et al. (2005) possibly detected chromatic microlensing and renewed interest in this lens system. The narrowband observations of Q 0142-100 were obtained at the NOT on two nights separated by 15 days (see Table 1). The best fit to the images was found using a de Vaucouleurs profile for the lensing galaxy, and point sources for the quasar images using the astrometry and structural models from Lehár et al. (2000).

Unfortunately, these observations shed little light on the chromatic behavior of Q 0142-100 (open squares in Figure 13). Because most of the NOT filters are again contaminated in varying amounts by the emission lines of the quasar. The most affected filters are the iac\#29 and Sty-b bands, which lie on the CIII] and Ly $\alpha$ emission lines. However, comparing our results with the data obtained by Elíasdóttir et al. (2006) (filled squares in Figure 13) and by CASTLES (Falco et al. 1999) (filled triangles), somewhat constrains the origin of the observed chromaticity. All three data sets, spanning a 10 year period, show the same color trends. Koptelova et al. (2010) also monitored this system during a 2 year period in $V$, $R$, and $I$ bands, and their average $m_{B}-m_{A}$ values for the different filters are consistent with our observations. This similarity between the color trends essentially eliminates the possibility that intrinsic source variability modulated by the time delay could produce the color trends, since the delay, while unknown, is small compared with the 10 year interval between epochs.

Extinction alone is also ruled out because the wavelengths of the observations correspond to the regime where all the extinction laws are roughly proportional to $\lambda$, with the $2175 \AA$ feature lying blueward of all the data, leaving nothing to create the observed parabolic 
wavelength dependence. Therefore the explanation for the observed chromaticity should be chromatic microlensing, even though the chromaticity changed little over a decade. Microlensing variations for Q 0142-100 are assured on the timescale $t_{E} \simeq 24$ years 2 it should take the source to cross an Einstein radius, although fluctuations can occur on the shorter timescale of $t_{S} \simeq 3$ years it should take to cross the optical source size 3 . This suggests that the images must be lying in the broad, relatively structureless "valleys" between the "active ridges" of the caustic networks. The redder color of image B may partly be due to contamination by the lens galaxy, since their separation is only 0.38 arcsec and our data are limited by the resolution of our ground-based observations.

\section{Summary and Conclusions}

In this work, we studied the chromatic behavior of four lens systems using optical multiwavelength data from a monitoring campaign performed at the NOT. The use of narrowband photometry under excellent seeing conditions has proved to be a powerful tool to disentangle chromatic microlensing from extinction, since depending on the source redshift, emission line contamination can be better separated from the continuum emission.

In particular, the color variations in SDSS J1650+4251 are probably dominated by extinction and require an extinction law without a $2175 \AA$ feature. This indicates that we have found a galaxy in which the extinction curve is similar to that of the SMC but at a cosmological redshift $\left(z_{L}=0.58\right)$. This result significantly increases the small sample of high-redshift galaxies with similar extinction features, and it is the first robustly determined from the pair method. This was possible due to the good wavelength resolution achieved by the use of narrow filters. The firm confirmation of an SMC-like dust at a cosmological distance is very important since many models including dust at higher redshifts assume the "featureless" SMC extinction law (e.g., Richards et al. 2003; Hopkins et al. 2004). The selection criteria would certainly be crucial in many fields of astrophysics, like, for instance, in the correct understanding of the expansion of the universe through SNe Ia (e.g., Jha et al. 2006, 2007).

\footnotetext{
${ }^{2}$ This timescale was calculated using a concordance cosmology $\left(\Omega_{m}=0.3, \Omega_{\Lambda}=0.7, H_{0}=72 \mathrm{~km} \mathrm{~s}-1\right.$ $\left.\mathrm{Mpc}^{-1}\right)$. The Einstein radius is $r_{E} \simeq 2.7 \times 10^{16} \mathrm{~cm}$ for $0.3 M_{\odot}$ microlenses, and the effective transverse velocity of the source (e.g., Kayser et al. 1986) is $v \approx 360 \mathrm{~km} \mathrm{~s}^{-1}$ for a velocity dispersion of $\sigma_{*}=237 \mathrm{~km}$ $\mathrm{s}^{-1}$.

${ }^{3}$ We estimated the V-band disk size assuming that the disk emits like a thin disk (Shakura \& Sunyaev 1973) and used the black hole mass of $M_{\mathrm{BH}}=2.26 \times 10^{9} M_{\odot}$ estimated by Peng et al. (2006). For these assumptions the disk size in the $\mathrm{V}$-band $\left(\lambda_{\text {rest }} \simeq 0.15 \mu \mathrm{m}\right)$ is $R_{V} \simeq 2.9 \times 10^{15} \mathrm{~cm}$.
} 
We clearly detect chromatic microlensing in the image A of HE 0435-1223. This single epoch observation, with microlensing $\Delta M_{R}=-0.19 \pm 0.04$ and chromatic microlensing $\Delta M_{b-R}=-0.13 \pm 0.08$, allows us to estimate the disk size and to constrain the power-law index in the size-wavelength scaling of the accretion disk. We found an R-band disk size of $r_{s}^{R}=13 \pm 5$ light days for a linear prior on $r_{s}^{R}$, and of $r_{s}^{R}=7 \pm 6$ light days for a logarithmic prior, and a value of $p=1.3 \pm 0.3$ consistent with the Shakura \& Sunyaev (1973) thin disk model. Our result is in good agreement with those of other authors (Pooley et al. 2007, 2009; Morgan et al. 2010; Blackburne et al. 2011).

In the case of FBQ $0951+2635$ and Q 0142-100, unfortunately, further observations are needed to completely understand the chromatic behavior of the systems, although our observations shed some light on their chromatic variations. The lack of chromaticity observed in the FBQ $0951+2635$ NOT data is compatible with the absence of extinction, and the different chromatic behaviors observed at different epochs suggest that there is significant microlensing in the system. In the case of Q 0142-100, microlensing scenarios are also favored to explain the observed chromaticity, although wavelength-dependent contamination by the lens galaxy in the flux of image B, due to its proximity, is not ruled out.

\section{Acknowledgments:}

This research was supported by the European Community's Sixth Framework Marie Curie Research Training Network Programme, Contract No. MRTN-CT-2004-505183 "ANGLES", and by the Spanish Ministerio de Educación y Ciencias (grants AYA2004-08243C03-01/03 and AYA2007-67342-C03-01/03). A.M.M. acknowledges the support of Gener-

alitat Valenciana, grant APOSTD/2010/030. J.A.M. is also supported by the Generalitat Valenciana with the grant PROMETEO/2009/64. C.S.K. is supported by NSF grants AST0708082 and AST-1009756. 


\section{REFERENCES}

Abajas, C., Mediavilla, E., Muñoz, J. A., Popović, L. C., \& Oscoz, A. 2002, ApJ, 576, 640

Anguita, T., Schmidt, R. W., Turner, E. L., Wambsganss, J., Webster, R. L., Loomis, K. A., Long, D., \& McMillan, R. 2008, A\&A, 480, 327

Assef, R. J., et al. 2010, ApJ, 713, 970

Bate, N. F., Floyd, D. J. E., Webster, R. L., \& Wyithe, J. S. B. 2008, MNRAS, 391, 1955

Bentz, M. C., Peterson, B. M., Netzer, H., Pogge, R. W., \& Vestergaard, M. 2009, ApJ, 697, 160

Bianchi, L., Clayton, G. C., Bohlin, R. C., Hutchings, J. B., \& Massey, P. 1996, ApJ, 471, 203

Blackburne, J. A., \& Kochanek, C. S. 2010, ApJ, 718, 1079

Blackburne, J. A., Pooley, D., Rappaport, S., \& Schechter, P. L. 2011, ApJ, in press (arXiv:1007.1665)

Blaes, O. M. 2004, in Accretion Discs, Jets and High Energy Phenomena in Astrophysics, Vol 78, ed. Vassily Beskin et al. (Berlin:Springer), 137

Cardelli, J. A., Clayton, G. C., \& Mathis, J.S. 1989, ApJ, 345, 245 (CCM)

Chartas, G., Kochanek, C. S., Dai, X., Poindexter, S., \& Garmire, G. 2009, ApJ, 693, 174

Courbin, F., et al. 2010, A\&A, submitted (arXiv:1009.1473)

Dai, X., \& Kochanek, C. S. 2009, ApJ, 692, 677

Dai, X., Kochanek, C. S., Chartas, G., Kozlowski, S., Morgan, C. W., Garmire, G., \& Agol, E. 2010, ApJ, 709, 278

Draine, B.T. 2003, ARA\&A, 41, 241

Eigenbrod, A., Courbin, F., \& Meylan, G. 2007, A\&A, 465, 51

Eigenbrod, A., Courbin, F., Meylan, G., Agol, E., Anguita, T., Schmidt, R. W., \& Wambsganss, J. 2008, A\&A, 490, 933

Elíasdóttir, Á., Hjorth, J., Toft, S., Burud, I., \& Paraficz, D. 2006, ApJS, 166, 443 
Elíasdóttir, Á., et al. 2009, ApJ, 697, 1725

Ellison, S. L., et al. 2006, MNRAS, 372, 38

Falco, E. E., et al. 1999, ApJ, 523, 617

Fitzpatrick, E. L., \& Massa, D. 1990, ApJS, 72, 163

Floyd, D. J. E., Bate, N. F., \& Webster, R. L. 2009, MNRAS, 398, 233

Gordon, K. D., Clayton, G.C., Misselt, K.A., Landolt, A.U., \& Wolff, M. J. 2003, ApJ, 594, 279

Hopkins, P. F., et al. 2004, AJ, 128, 1112

Jakobsson, P., Hjorth, J., Burud, I., Letawe, G., Lidman, C., \& Courbin, F. 2005, A\&A, 431, 103

Jakobsson, P., et al. 2004, A\&A, 427, 785

Jean, C., \& Surdej, J. 1998, A\&A, 339, 729

Jha, S., Riess, A. G., \& Kirshner, R. P. 2007, ApJ, 659, 122

Jha, S., et al. 2006, AJ, 131, 527

Junkkarinen, V. T., Cohen, R. D., Beaver, E. A., Burbidge, E. M., Lyons, R.W., \& Madejski, G. 2004, ApJ, 614, 658

Kann, D. A., Klose, S., \& Zeh, A. 2006, ApJ, 641, 993

Kayser, R., Refsdal, S., \& Stabell, R. 1986, A\&A, 166, 36

Keeton, C. 2001, arXiv:astro-ph/0102340

Kochanek, C. S. 2006, in Saas-Fee Advanced Course 33, Gravitational Lensing: Strong, Weak and Micro, ed. G. Meylan, P. Jetzer, \& P. North (Berlin: Springer), 91

Kochanek, C. S., Morgan, N. D., Falco, E. E., McLeod, B. A., Winn, J. N., Dembicky, J., \& Ketzeback, B. 2006, ApJ, 640, 47

Kochanek, C. S., et al. 2000, ApJ, 543, 131

Koptelova, E., Oknyanskij, V. L., Artamonov, B. P., \& Burkhonov, O. 2010, MNRAS, 401, 2805 
Lehár, J., et al. 2000, ApJ, 536, 584

MacAlpine, G. M. \& Feldman, F. R. 1982, ApJ, 261, 412

Massa, D., Savage, B. D., \& Fitzpatrick, E. L. 1983, ApJ, 266, 662

McLeod, B. A., Bernstein, G. M., Rieke, M. J., \& Weedman, D. W. 1998, AJ, 115, 1377

Mediavilla, E., Muñoz, J. A., Kochanek, C. S., Falco, E. E., Arribas, S., \& Motta, V. 2005, ApJ, 619, 749

Mediavilla, E., Muoz, J. A., Lopez, P., Mediavilla, T., Abajas, C., Gonzalez-Morcillo, C., \& Gil-Merino, R. 2006, ApJ, 653, 942

Mediavilla, E., et al. 2009, ApJ, 706, 1451

Ménard, B., Nestor, D., Turnshek, D., Quider, A., Richards, G., Chelouche, D., \& Rao, S. 2008, MNRAS, 385, 1053

Misselt, K. A., Clayton, G. C., \& Gordon, K. D. 1999, ApJ, 515, 128

Morgan, C. W., Kochanek, C. S., Dai, X., Morgan, N. D., \& Falco, E. E. 2008, ApJ, 689, 755

Morgan, C. W., Kochanek, C. S., Morgan, N. D., \& Falco, E. E. 2010, ApJ, 712, 1129

Morgan, N. D., Kochanek, C. S., Pevunova, O., \& Schechter, P. L. 2005, AJ, 129, 2531

Morgan, N. D., Snyder, J. A., \& Reens, L. H. 2003, AJ, 126, 2145

Mosquera, A. M., Muñoz, J. A., \& Mediavilla, E. 2009, ApJ, 691, 1292

Motta, V., et al. 2002, ApJ, 574, 719

Muñoz, J. A., Falco, E. E., Kochanek, C. S., McLeod, B. A., \& Mediavilla, E. 2004, ApJ, 605,614

Murphy, M. T., \& Liske, J. 2004, MNRAS, 354, 31

Nadeau, D., Yee, H. K. C., Forrest, W. J., Garnett, J. D., Ninkov, Z., \& Pipher, J. L. 1991, ApJ, 376, 430

Nakos, Th., et al. 2005, A\&A, 441, 443

Nandy, K., Morgan, D. H., Willis, A. J., Wilson, R., \& Gondhalekar, P. M. 1981, MNRAS, 196,955 
Perlmutter, S., et al. 1997, ApJ, 483, 565

Peng, C. Y., Impey, C. D., Rix, H. W., Kochanek, C. S., Keeton, C. R., Falco, E. E., Lehr, J., \& McLeod, B. A., 2006, ApJ, 649, 616

Poindexter, S., Morgan, N., \& Kochanek, C. S. 2008, ApJ, 673, 34

Pooley, D., Blackburne, J. A., Rappaport, S., \& Schechter, P. L. 2007, ApJ, 661, 19

Pooley, D., Rappaport, S., Blackburne, J., Schechter, P. L., Schwab, J., \& Wambsganss, J. 2009, ApJ, 697, 1892

Prèvot, M. L., Lequeux, J., Maurice, E., Prèvot, L., \& Rocca-Volmerange, B. 1984, A\&A, 132, 389

Richards, G. T., et al. 2001, AJ, 121, 2308

Richards, G. T., et al. 2003, AJ, 126, 1131

Riess, A. G., Press, W. H., \& Kirshner, R. P. 1996, ApJ, 473, 88

Schechter, P. L., Gregg, M. D., Becker, R. H., Helfand, D. J., \& White, R. L. 1998, AJ, 115, 1371

Shakura, N. I., \& Sunyaev, R. A. 1973, A\&A, 24, 337

Surdej, J., Swings, J.-P., Magain, P., Courvoisier, T. J.-L., \& Borgeest, U. 1987, Nature, 329,695

Vanden Berk, D. E., et al. 2001, AJ, 122, 549

Vuissoz, C., et al. 2007, A\&A, 464, 845

Wambsganss, J. 2006, in Saas-Fee Advanced Course 33, Gravitational Lensing: Strong, Weak and Micro, ed. G. Meylan, P. Jetzer, \& P. North (Berlin: Springer), 453

Wambsganss, J., \& Paczynski, B. 1991, AJ, 102, 864

Wang, J., Hall, P. B., Ge, J., Li, A., \& Schneider, D. P. 2004, ApJ, 609, 589

Wild, V., Hewett, P. C., \& Pettini, M., 2006, MNRAS, 367, 211

Wisotzki, L., Becker, T., Christensen, L., Helms, A., Jahnke, K., Kelz, A., Roth, M. M., \& Sanchez, S. F. 2003, A\&A, 408, 455 
Wisotzki, L., Christlieb, N., Bade, N., Beckmann, V., Khler, T., Vanelle, C., \& Reimers, D. 2000, A\&A, 358, 77

Wisotzki, L., Schechter, P. L., Bradt, H. V., Heinmller, J., \& Reimers, D. 2002, A\&A, 395, 17

Wisotzki, L., et al. 2004, Astron. Nachr., 325, 135 


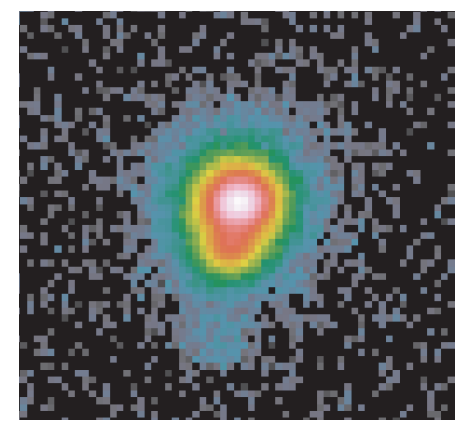

SDSS J1650+4251

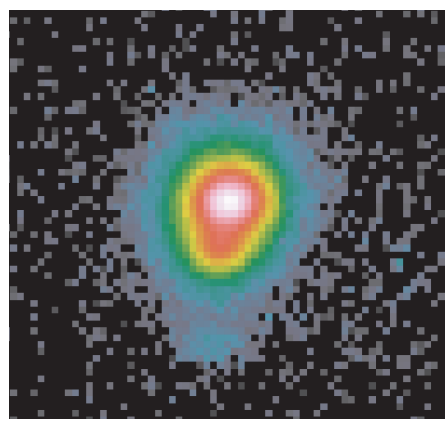

Modelled image

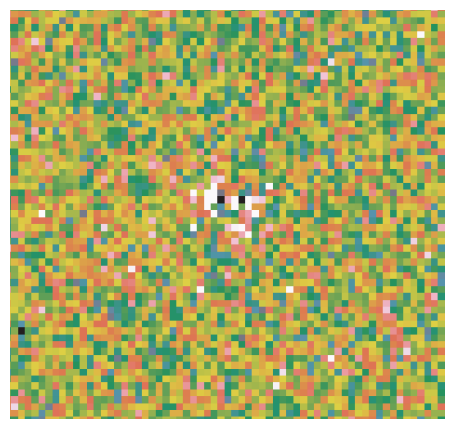

Residuals

Fig. 1. - Str-y image (left) of SDSS J1650+4251, our photometric model consisting of two point sources (middle), and the residuals (right) after subtracting the model from the data. The peak residuals (white) are roughly $2 \%$ of the quasar peak. 


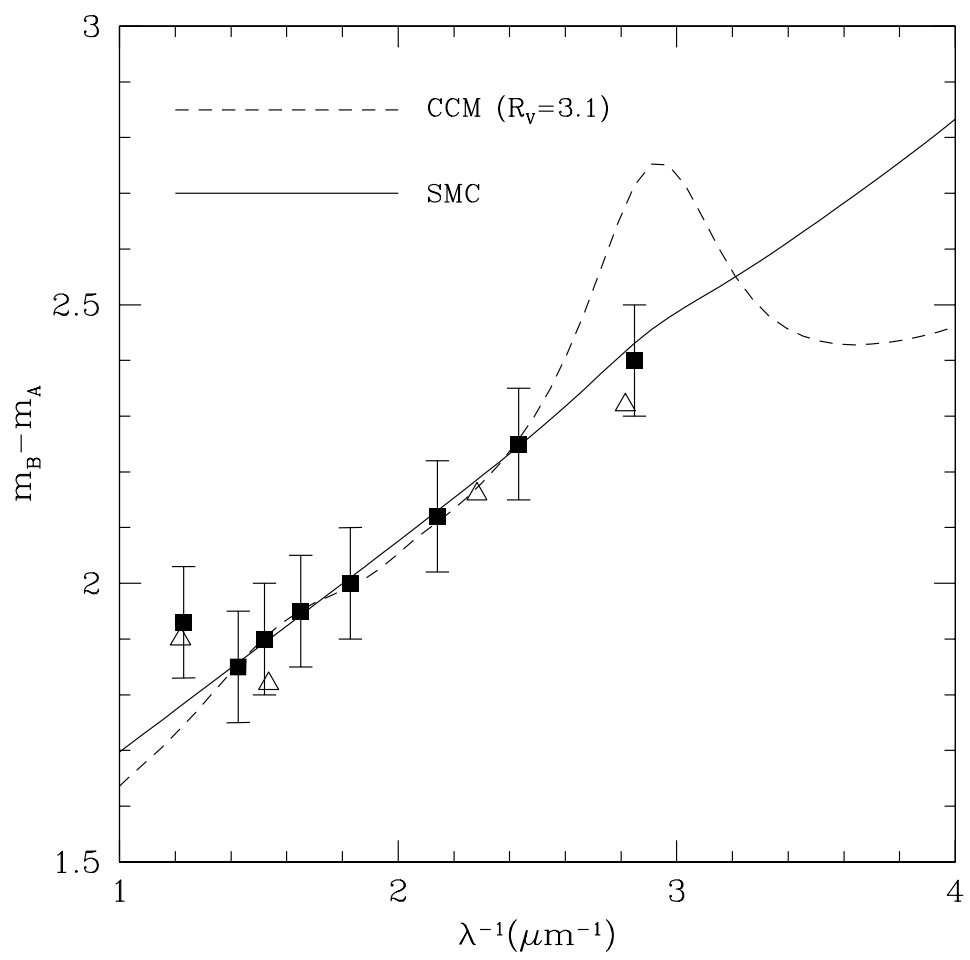

Fig. 2.- Difference in magnitude vs. the inverse of the observed wavelength for the quasar images of the lens system SDSS J1650+4251. The filled squares correspond to our NOT data and the open triangles to those obtained by Morgan et al. (2003). The variation in the magnitude difference with wavelength is probably produced by differential extinction in the lens galaxy. An extinction law similar to the SMC fits the observations well, while a Milky Way extinction law (CCM) is ruled out. 


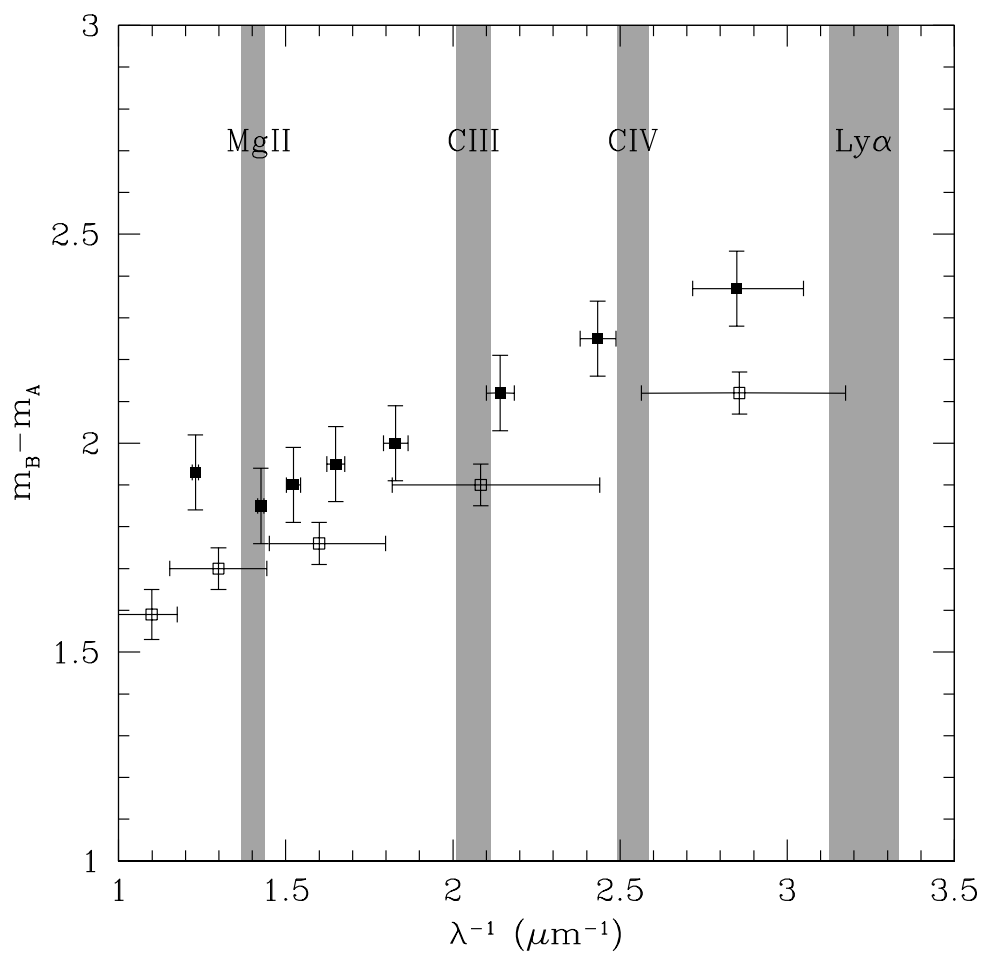

Fig. 3.- Difference in magnitude vs. the inverse of the observed wavelength for the quasar images of the lens system SDSS $1650+4251$. The filled squares correspond to the data taken at the NOT (2003) and the open squares correspond to data taken at the LT (2006). The trend with wavelength is the same in all the different epochs, although a magnitude shift due to microlensing may also be present. The wavelength regions corresponding to the most prominent quasar emission lines are indicated (from left to right: MgII, CIII, and CIV ). The horizontal error bars correspond to the filter widths. 


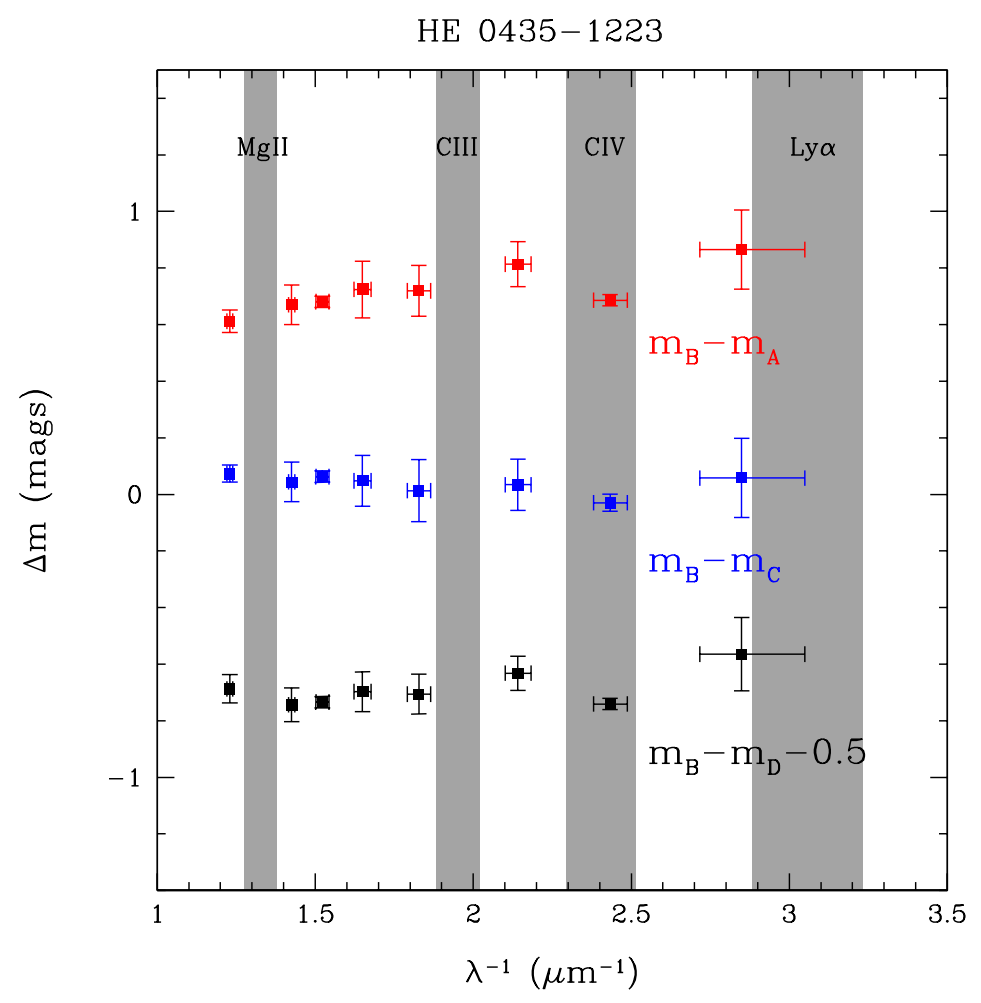

Fig. 4.- Magnitude differences as a function of the inverse of the observed wavelength for HE 0435-1223 (NOT data on HJD 2454404). Images A and D show a clear signature of microlensing in the continuum but not in the emission lines. Moreover, the chromatic variation observed in image A could only be explained as a consequence of chromatic microlensing. The wavelength regions corresponding to the most prominent quasar emission lines are indicated (from left to right: MgII, CIII, CIV and Ly $\alpha$ ). The horizontal error bars correspond to the filter widths. 


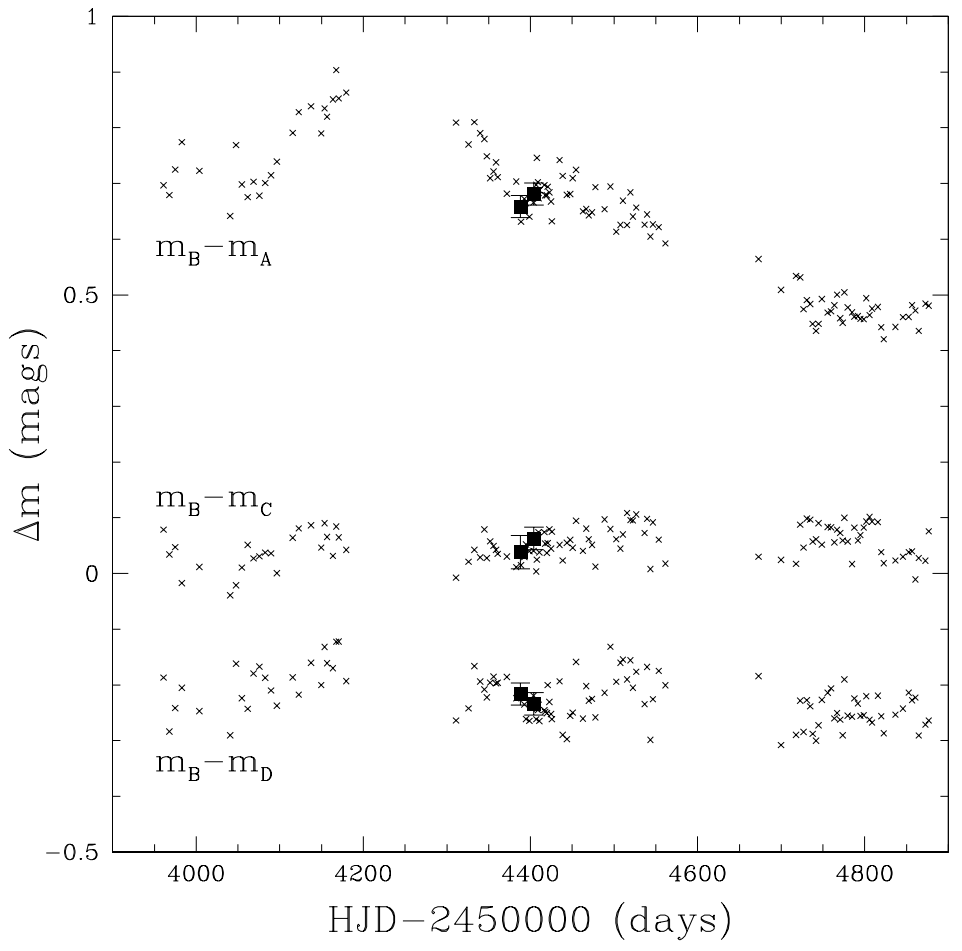

Fig. 5.- HE 0435-1223 SMARTS R-band light curve (Blackburne \& Kochanek 2010) corrected for time delays. The filled squares correspond to our observation in the redshift zero $\mathrm{H} \alpha$ filter, which is a good match to the R-band wavelength. 


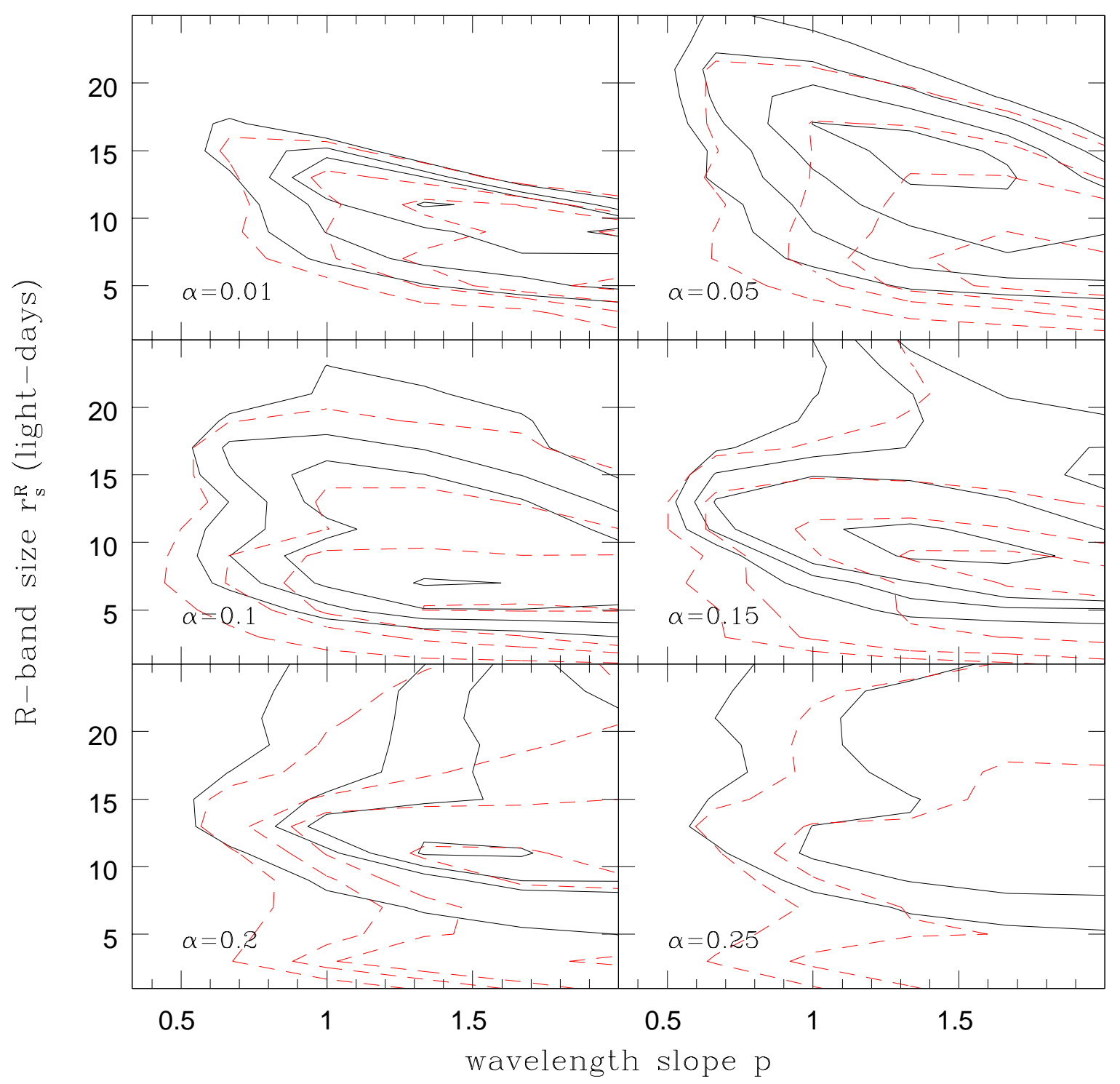

Fig. 6.- Probability of reproducing the observed microlensing magnification and chromaticity of HE $0435-1223$ as a function of the R-band disk size, $r_{s}^{R}$, and the power-law index, $p$, where $r_{s}^{R} \propto \lambda^{p}$. The panels show the results for different stellar mass fractions $\alpha$. The contours correspond to $15 \%, 47 \%, 68 \%$, and $90 \%$ confidence intervals. Uniform (solid line) and logarithmic (dashed line) priors on $r_{s}^{R}$ were assumed. 

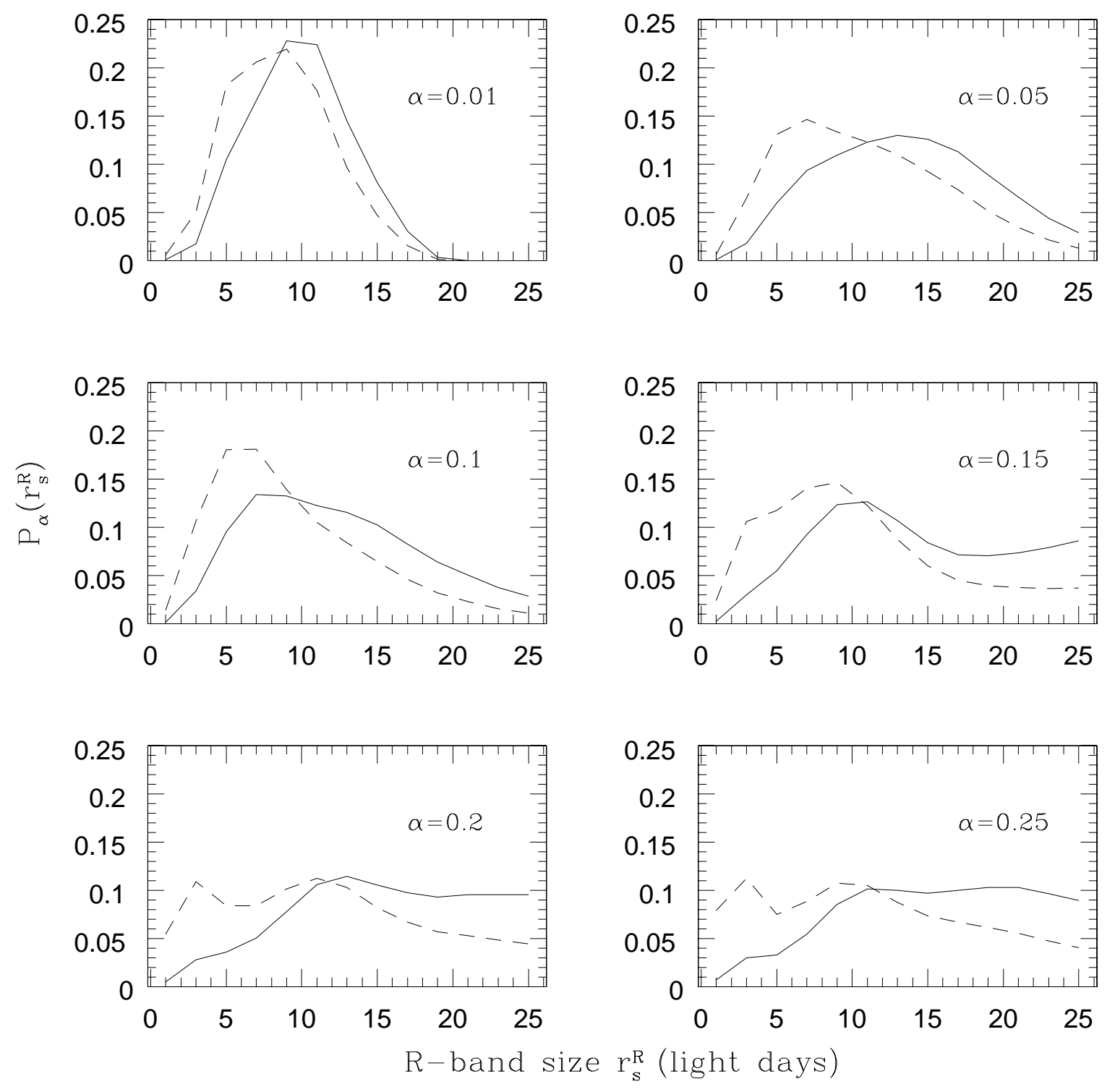

Fig. 7.- Probability of reproducing the observed microlensing magnification and chromaticity of HE $0435-1223$ as a function of the R-band disk size, $r_{s}^{R}$ for different stellar mass fractions $\alpha$, and normalized by the total probability in the $\left(r_{s}^{R}, p\right)$ grid. Uniform (solid line) and logarithmic (dashed line) priors on $r_{s}^{R}$ were assumed. 

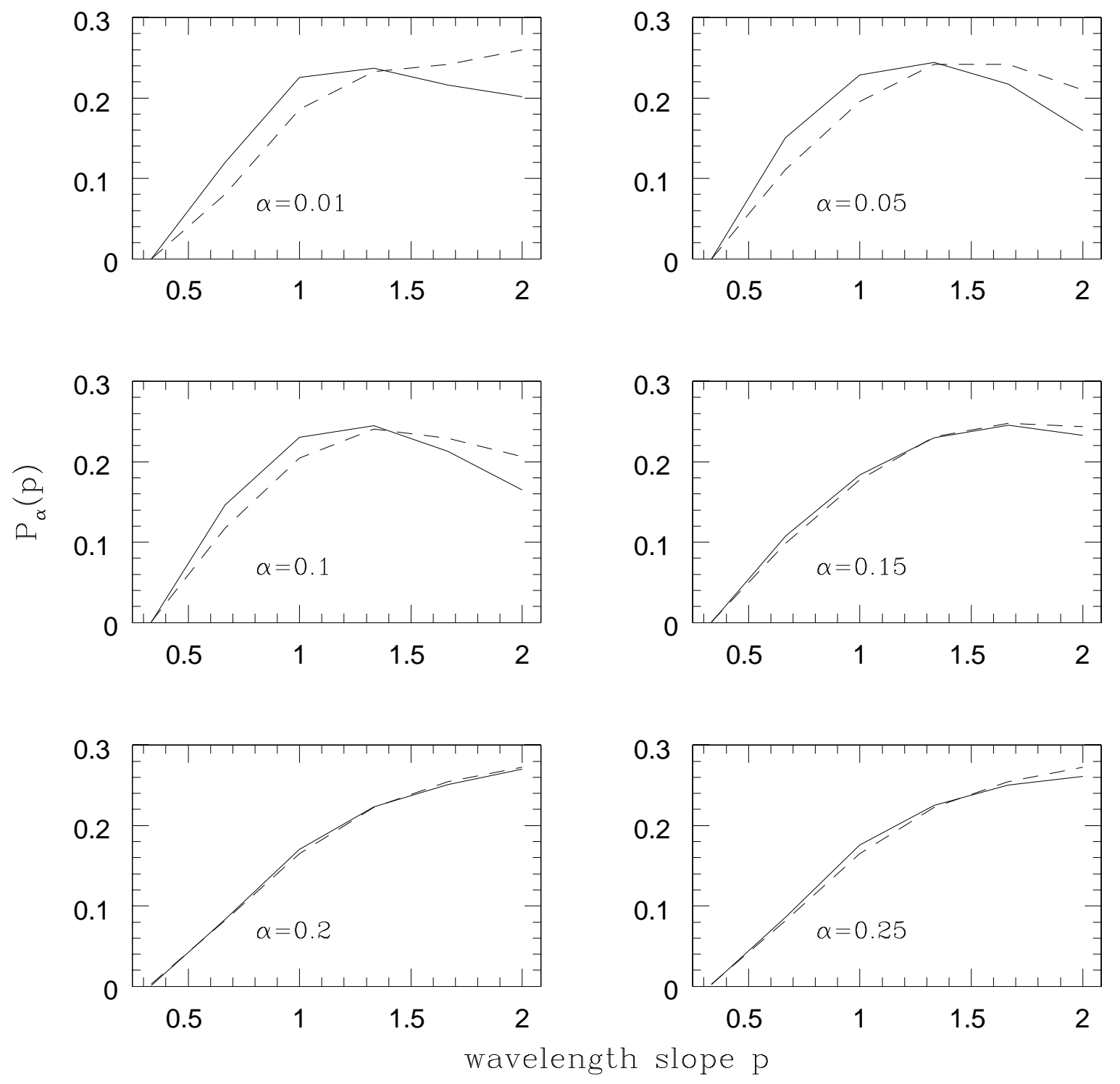

Fig. 8.- Probability of reproducing the observed microlensing magnification and chromaticity of HE $0435-1223$ as a function of the power-law index, $p$ for different stellar mass fractions $\alpha$, and normalized by the total probability in the $\left(r_{s}^{R}, p\right)$ grid. Uniform (solid line) and logarithmic (dashed line) priors on $r_{s}^{R}$ were assumed. 


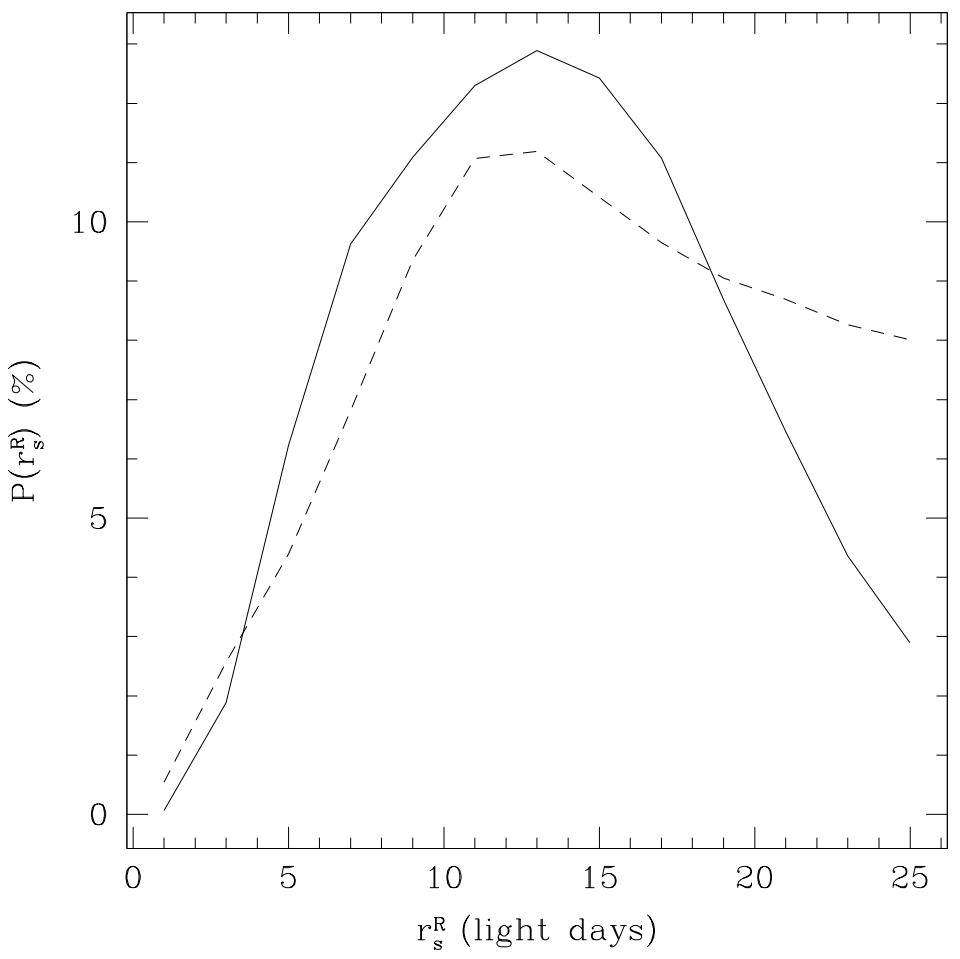

Fig. 9.- Probability of reproducing the observed microlensing magnification and chromaticity in HE $0435-1223$ as a function of the R-band disk size, $r_{s}^{R}$, after marginalizing over $\alpha$. The solid line was obtained using the prior on $\alpha$ from Mediavilla et al. (2009). The dashed line corresponds to the probability distribution without this prior. These results are for the uniform prior on $r_{s}^{R}$. 


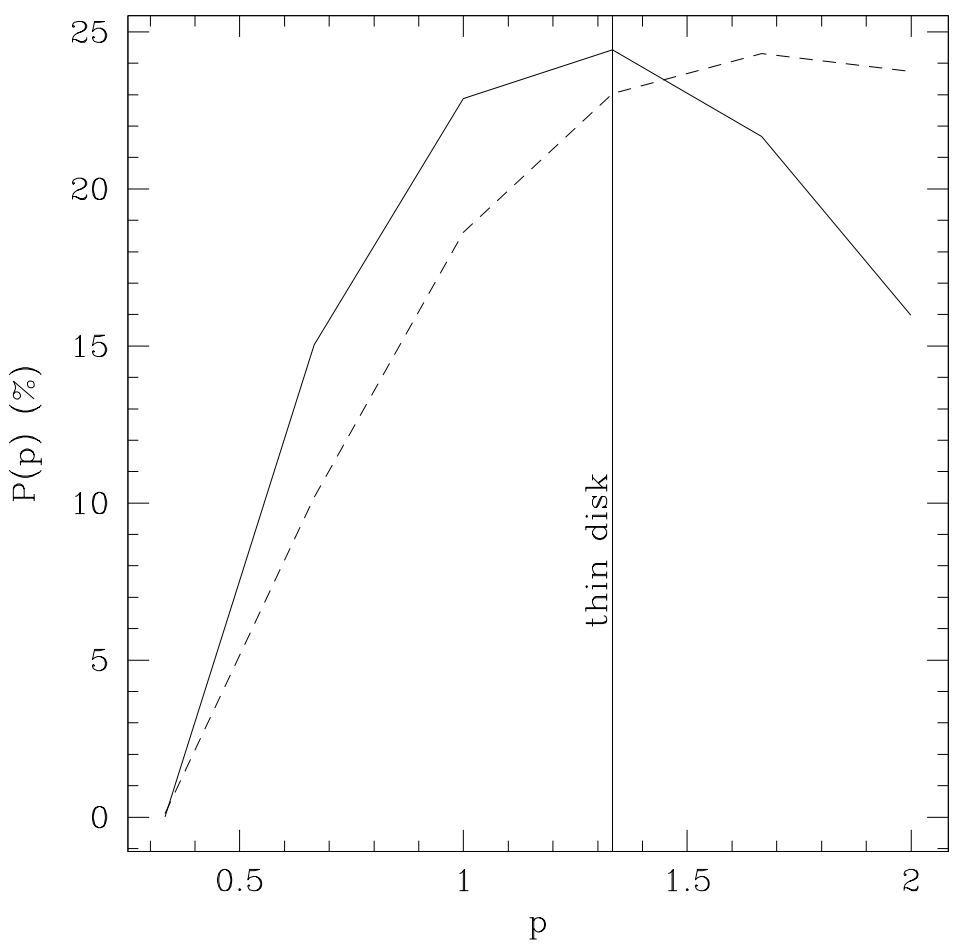

Fig. 10.- Probability of reproducing the observed microlensing magnification and chromaticity in HE 0435-1223 as a function of the power-law index, $p$, after marginalizing over $\alpha$. The solid line was obtained using the prior on $\alpha$ from Mediavilla et al. (2009). The dashed line corresponds to the probability distribution without this prior. These results are for the uniform prior on $r_{s}^{R}$. 


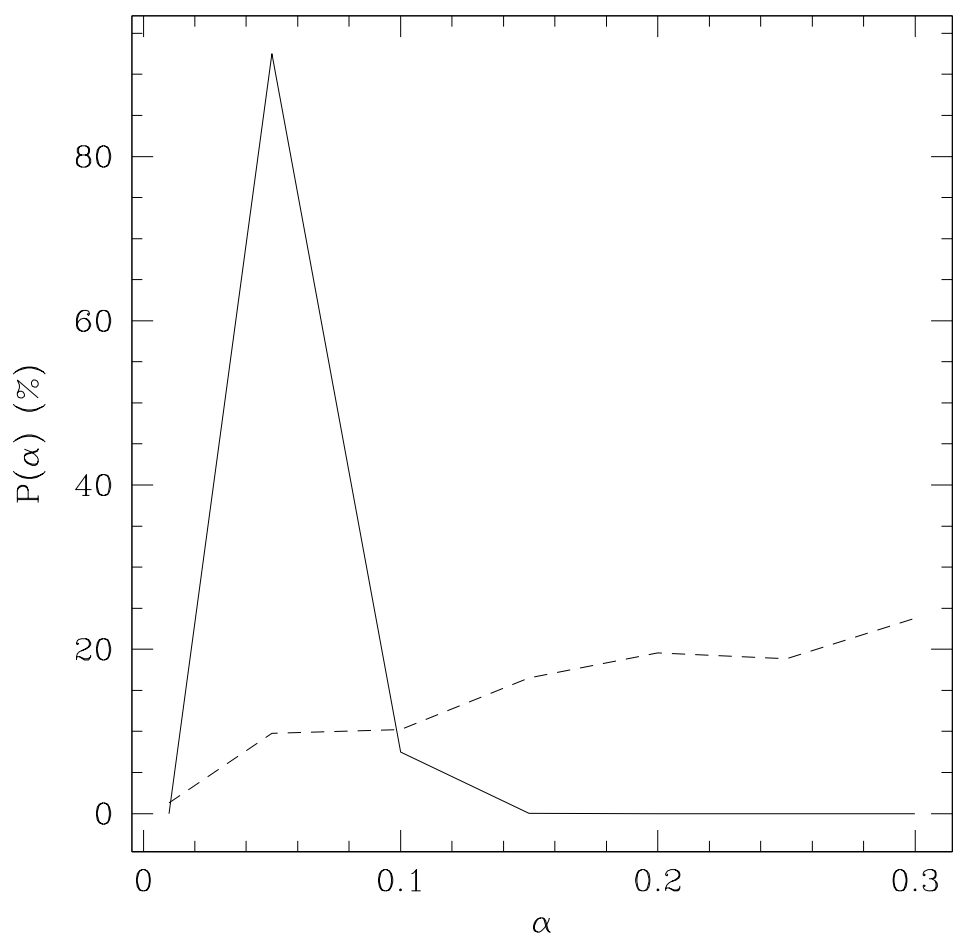

Fig. 11. - Probability of reproducing the observed microlensing magnification and chromaticity HE $0435-1223$ as a function of the stellar fraction, $\alpha$, marginalized over the R-band disk size $r_{s}^{R}$ and the power-law index $p$. The solid line was obtained using the prior on $\alpha$ from Mediavilla et al. (2009). The dashed line corresponds to the probability distribution without this prior. These results are for the uniform prior on $r_{s}^{R}$. 


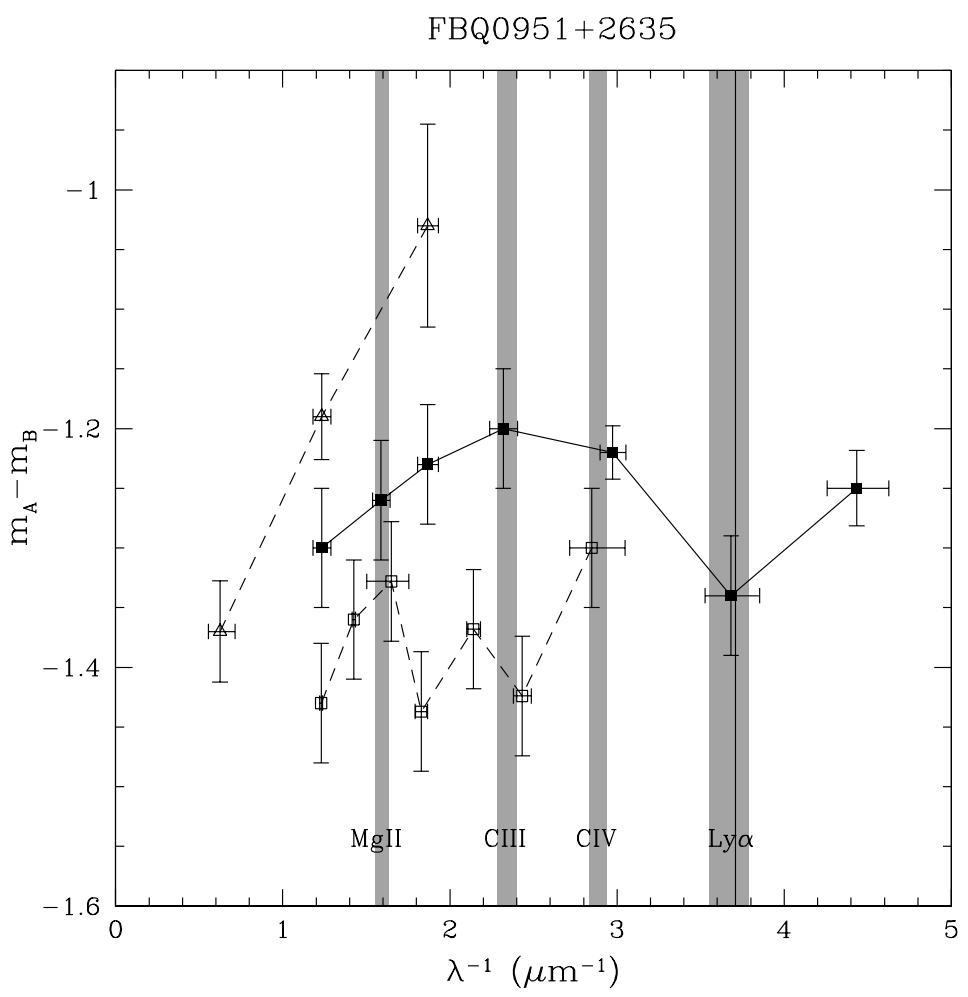

Fig. 12.- Magnitude differences as a function of the inverse of the observed wavelength for FBQ 0951+2635. The NOT observations (open squares) are compared with the CASTLES (open triangles) and J. A. Muñoz et al. (2011, in preparation, filled squares) HST observations. The wavelengths corresponding to the most prominent quasar emission lines are indicated (from left to right: MgII, CIII, CIV and Ly $\alpha$ ). The horizontal error bars correspond to the filter widths. The solid vertical line in the Ly $\alpha$ band corresponds to the expected position of the $2175 \AA$ feature. 


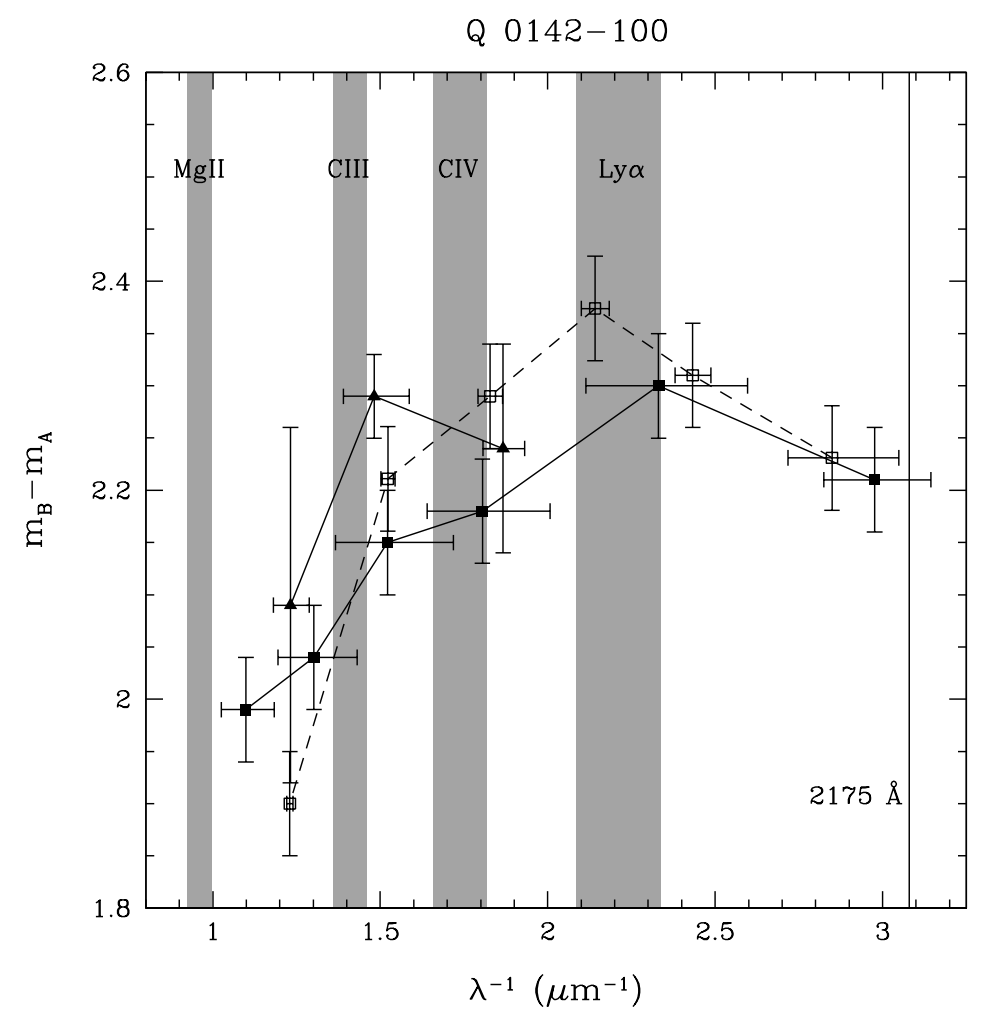

Fig. 13. - Magnitude differences as a function of the inverse of the observed wavelength for Q 0142-100 at different epochs. The open squares correspond to the NOT data. The filled squares correspond to the data from Elíasdóttir et al. (2006), and the filled triangles are the data from CASTLES. The wavelengths corresponding to the most prominent quasar emission lines are indicated (from left to right: MgII, CIII], CIV and Ly $\alpha$ ). The horizontal error bars correspond to the filter widths. The solid vertical line corresponds to the expected position of the $2175 \AA$ feature. 
Table 1. $\quad \log$ of ALSFOC Observations

\begin{tabular}{|c|c|c|c|}
\hline Target & Observation Date & Filter & Exposure (s) \\
\hline SDSS J1650+4251 & 2003 Aug 26 & $\operatorname{Str}-\mathrm{u}(\lambda=3510 \AA)$ & $4 \times 300$ \\
\hline SDSS J1650+4251 & 2003 Aug 26 & I-band $(\lambda=8130 \AA)$ & $3 \times 900$ \\
\hline SDSS J1650+4251 & 2003 Aug 28 & $\operatorname{Str}-\mathrm{b}(\lambda=4670 \AA)$ & $3 \times 600$ \\
\hline SDSS J1650+4251 & 2003 Aug 28 & $\operatorname{Str}-y(\lambda=5470 \AA)$ & $3 \times 600$ \\
\hline SDSS J1650+4251 & 2003 Aug 28 & $\operatorname{Iac\# 28}(\lambda=6062 \AA)$ & $3 \times 600$ \\
\hline SDSS J1650+4251 & 2003 Aug 28 & $\mathrm{H} \alpha(\lambda=6567 \AA)$ & $3 \times 600$ \\
\hline SDSS J1650+4251 & 2003 Aug 28 & Iac\#29 $(\lambda=7015 \AA)$ & $3 \times 600$ \\
\hline SDSS J1650+4251 & 2003 Aug 28 & I-band $(\lambda=8130 \AA)$ & $1 \times 300$ \\
\hline HE $0435-1223$ & 2007 Oct 15 & $\operatorname{Str}-\mathrm{v}(\lambda=4110 \AA)$ & $3 \times 600$ \\
\hline HE $0435-1223$ & 2007 Oct 15 & Str-y $(\lambda=5470 \AA)$ & $3 \times 600$ \\
\hline HE $0435-1223$ & 2007 Oct 15 & $\mathrm{H} \alpha(\lambda=6567 \AA)$ & $3 \times 600$ \\
\hline HE $0435-1223$ & 2007 Oct 15 & I-band $(\lambda=8130 \AA)$ & $4 \times 100$ \\
\hline HE $0435-1223$ & 2007 Oct 31 & $\operatorname{Str}-\mathrm{u}(\lambda=3510 \AA)$ & $4 \times 600$ \\
\hline HE $0435-1223$ & 2007 Oct 31 & $\operatorname{Str}-\mathrm{v}(\lambda=4110 \AA)$ & $3 \times 600$ \\
\hline HE $0435-1223$ & 2007 Oct 31 & $\operatorname{Str}-\mathrm{b}(\lambda=4670 \AA)$ & $3 \times 600$ \\
\hline HE $0435-1223$ & 2007 Oct 31 & $\operatorname{Str}-y(\lambda=5470 \AA)$ & $3 \times 600$ \\
\hline HE $0435-1223$ & 2007 Oct 31 & $\operatorname{Iac} \# 28(\lambda=6062 \AA)$ & $3 \times 600$ \\
\hline HE $0435-1223$ & 2007 Oct 31 & $\mathrm{H} \alpha(\lambda=6567 \AA)$ & $3 \times 600$ \\
\hline HE $0435-1223$ & 2007 Oct 31 & Iac\#29 $(\lambda=7015 \AA)$ & $3 \times 600$ \\
\hline HE $0435-1223$ & 2007 Oct 31 & I-band $(\lambda=8130 \AA)$ & $4 \times 100$ \\
\hline FBQ $0951+2635$ & 2006 Nov 17 & $\operatorname{Str}-\mathrm{u}(\lambda=3510 \AA)$ & $4 \times 600$ \\
\hline FBQ $0951+2635$ & 2006 Nov 17 & Str-v $(\lambda=4110 \AA)$ & $3 \times 600$ \\
\hline FBQ $0951+2635$ & 2006 Nov 17 & $\operatorname{Str}-\mathrm{b}(\lambda=4670 \AA)$ & $3 \times 600$ \\
\hline FBQ $0951+2635$ & 2006 Nov 17 & $\operatorname{Str}-y(\lambda=5470 \AA)$ & $3 \times 600$ \\
\hline FBQ $0951+2635$ & 2006 Nov 17 & $\operatorname{Iac\# 28}(\lambda=6062 \AA)$ & $3 \times 600$ \\
\hline FBQ $0951+2635$ & 2006 Nov 17 & $\operatorname{Iac} \# 29(\lambda=7015 \AA)$ & $3 \times 600$ \\
\hline FBQ $0951+2635$ & 2006 Nov 17 & I-band $(\lambda=8130 \AA)$ & $4 \times 100$ \\
\hline Q $0142-100$ & 2006 Sep 23 & $\operatorname{Str}-\mathrm{u}(\lambda=3510 \AA)$ & $4 \times 600$ \\
\hline Q $0142-100$ & 2006 Sep 23 & $\operatorname{Str}-\mathrm{v}(\lambda=4110 \AA)$ & $3 \times 600$ \\
\hline Q $0142-100$ & 2006 Sep 23 & $\operatorname{Str}-\mathrm{b}(\lambda=4670 \AA)$ & $3 \times 600$ \\
\hline Q $0142-100$ & 2006 Sep 23 & $\operatorname{Str}-y(\lambda=5470 \AA)$ & $3 \times 600$ \\
\hline Q $0142-100$ & 2006 Sep 23 & $\mathrm{H} \alpha(\lambda=6567 \AA)$ & $3 \times 600$ \\
\hline Q $0142-100$ & 2006 Sep 23 & I-band $(\lambda=8130 \AA)$ & $4 \times 100$ \\
\hline Q $0142-100$ & 2006 Nov 17 & $\operatorname{Str}-\mathrm{u}(\lambda=3510 \AA)$ & $4 \times 600$ \\
\hline Q $0142-100$ & 2006 Nov 17 & $\operatorname{Str}-\mathrm{b}(\lambda=4670 \AA)$ & $3 \times 600$ \\
\hline Q $0142-100$ & 2006 Nov 17 & $\operatorname{Iac} \# 28(\lambda=6062 \AA)$ & $3 \times 600$ \\
\hline Q $0142-100$ & 2006 Nov 17 & $\operatorname{Iac} \# 29(\lambda=7015 \AA)$ & $3 \times 600$ \\
\hline Q $0142-100$ & 2006 Nov 17 & I-band $(\lambda=8130 \AA)$ & $4 \times 100$ \\
\hline
\end{tabular}

Str is short for Strömgren.

Iac\#28 and Iac\#29 are non-standard filters shared with the IAC-80 telescope (Observatorio del Teide, Tenerife, Spain). These two filters have an FWHM of 20 $\mathrm{nm}$ and $9.5 \mathrm{~nm}$, respectively. 
Table 2. $\quad \log$ of RATCam Observations

\begin{tabular}{|c|c|c|c|}
\hline Target & Observation Date & Filter & Exposure (s) \\
\hline SDSS J1650+4251 & 2006 Jun 14 & $\mathrm{u}^{\prime}(\lambda=3500 \AA)$ & $7 \times 120$ \\
\hline SDSS J1650+4251 & 2006 Jun 14 & $\mathrm{~g}^{\prime}(\lambda=4800 \AA)$ & $4 \times 100$ \\
\hline SDSS J1650+4251 & 2006 Jun 14 & $\mathrm{r}^{\prime}(\lambda=6250 \AA)$ & $2 \times 100$ \\
\hline SDSS J1650+4251 & 2006 Jun 14 & i' $(\lambda=7700 \AA)$ & $2 \times 100$ \\
\hline SDSS J1650+4251 & 2006 Jun 14 & $\mathrm{z}^{\prime}(\lambda=9100 \AA)$ & $3 \times 120$ \\
\hline SDSS J1650+4251 & 2006 Jun 24 & $\mathrm{u}^{\prime}(\lambda=3500 \AA)$ & $3 \times 120$ \\
\hline SDSS J1650+4251 & 2006 Jun 24 & $\mathrm{~g}^{\prime}(\lambda=4800 \AA)$ & $3 \times 100$ \\
\hline SDSS J1650+4251 & 2006 Jun 24 & $\mathrm{r}^{\prime}(\lambda=6250 \AA)$ & $2 \times 100$ \\
\hline SDSS J1650+4251 & 2006 Jun 24 & i' $(\lambda=7700 \AA)$ & $1 \times 100$ \\
\hline SDSS J1650+4251 & 2006 Jun 24 & $\mathrm{z}^{\prime}(\lambda=9100 \AA)$ & $3 \times 120$ \\
\hline SDSS J1650+4251 & 2006 Jun 30 & $\mathrm{u}^{\prime}(\lambda=3500 \AA)$ & $1 \times 120$ \\
\hline SDSS J1650+4251 & 2006 Jun 30 & $\mathrm{~g}^{\prime}(\lambda=4800 \AA)$ & $2 \times 100$ \\
\hline SDSS J1650+4251 & 2006 Jun 30 & $\mathrm{r}^{\prime}(\lambda=6250 \AA)$ & $1 \times 100$ \\
\hline SDSS J1650+4251 & 2006 Jun 30 & i' $(\lambda=7700 \AA)$ & $5 \times 100$ \\
\hline SDSS J1650+4251 & 2006 Jul 7 & $\mathrm{u}^{\prime}(\lambda=3500 \AA)$ & $3 \times 120$ \\
\hline SDSS J1650+4251 & $2006 \mathrm{Jul} 7$ & $\mathrm{~g}^{\prime}(\lambda=4800 \AA)$ & $5 \times 100$ \\
\hline SDSS J1650+4251 & $2006 \mathrm{Jul} 7$ & $\mathrm{r}^{\prime}(\lambda=6250 \AA)$ & $4 \times 100$ \\
\hline SDSS J1650+4251 & $2006 \mathrm{Jul} 7$ & i' $(\lambda=7700 \AA)$ & $4 \times 100$ \\
\hline SDSS J1650+4251 & $2006 \mathrm{Jul} 7$ & $\mathrm{z}^{\prime}(\lambda=9100 \AA)$ & $4 \times 120$ \\
\hline
\end{tabular}

Table 3. SDSS 1650+4251 LT PHOTOMETRY

\begin{tabular}{cc}
\hline \hline Filter & $m_{B}-m_{A}$ \\
\hline $\mathrm{u}^{\prime}(\lambda=3500 \AA)$ & $2.12 \pm 0.05$ \\
$\mathrm{~g}^{\prime}(\lambda=4800 \AA)$ & $1.90 \pm 0.05$ \\
$\mathrm{r}^{\prime}(\lambda=6250 \AA)$ & $1.76 \pm 0.05$ \\
$\mathrm{i}^{\prime}(\lambda=7700 \AA)$ & $1.70 \pm 0.05$ \\
$\mathrm{z}^{\prime}(\lambda=9100 \AA)$ & $1.59 \pm 0.06$ \\
\hline
\end{tabular}


Table 4. NOT-PнотометRY

\begin{tabular}{|c|c|c|c|c|c|c|c|c|c|}
\hline Target & $\Delta m$ & Str-u & Str-v & Str-b & Str-y & Iac\#28 & $\mathrm{H} \alpha$ & Iac\#29 & I-band \\
\hline SDSS $1650+4251$ & $m_{B}-m_{A}$ & $2.37 \pm 0.09$ & $2.25 \pm 0.09$ & $2.12 \pm 0.09$ & $2.00 \pm 0.09$ & $1.95 \pm 0.09$ & $1.90 \pm 0.09$ & $1.85 \pm 0.09$ & $1.93 \pm 0.09$ \\
\hline \multirow{3}{*}{ HE $0435-1223^{*}$} & $m_{B}-m_{A}$ & & $0.65 \pm 0.02$ & & $0.71 \pm 0.01$ & & $0.66 \pm 0.02$ & & $0.61 \pm 0.05$ \\
\hline & $m_{B}-m_{C}$ & & $-0.01 \pm 0.03$ & & $0.01 \pm 0.01$ & & $0.04 \pm 0.03$ & & $0.07 \pm 0.05$ \\
\hline & $m_{B}-m_{D}$ & & $0.15 \pm 0.03$ & & $0.16 \pm 0.01$ & & $0.22 \pm 0.02$ & & $0.22 \pm 0.04$ \\
\hline \multirow[t]{3}{*}{ HE $0435-1223^{* *}$} & $m_{B}-m_{A}$ & $0.87 \pm 0.14$ & $0.69 \pm 0.02$ & $0.81 \pm 0.08$ & $0.72 \pm 0.09$ & $0.72 \pm 0.10$ & $0.68 \pm 0.02$ & $0.67 \pm 0.07$ & $0.61 \pm 0.04$ \\
\hline & $m_{B}-m_{C}$ & $0.06 \pm 0.14$ & $-0.03 \pm 0.03$ & $0.03 \pm 0.09$ & $0.01 \pm 0.11$ & $0.05 \pm 0.09$ & $0.06 \pm 0.02$ & $0.04 \pm 0.07$ & $0.07 \pm 0.03$ \\
\hline & $m_{B}-m_{D}$ & $-0.06 \pm 0.13$ & $-0.24 \pm 0.02$ & $-0.13 \pm 0.06$ & $-0.21 \pm 0.07$ & $-0.20 \pm 0.07$ & $-0.23 \pm 0.02$ & $-0.24 \pm 0.06$ & $-0.19 \pm 0.05$ \\
\hline FBQ $0951+2635$ & $m_{B}-m_{A}$ & $1.30 \pm 0.05$ & $1.42 \pm 0.05$ & $1.37 \pm 0.05$ & $1.44 \pm 0.05$ & $1.33 \pm 0.05$ & & $1.36 \pm 0.05$ & $1.43 \pm 0.05$ \\
\hline Q $0142-100^{\dagger}$ & $m_{B}-m_{A}$ & $2.23 \pm 0.05$ & $2.31 \pm 0.05$ & $2.37 \pm 0.05$ & $2.29 \pm 0.05$ & & $2.21 \pm 0.05$ & & $2.39 \pm 0.05$ \\
\hline Q $0142-100{ }^{\dagger \dagger}$ & $m_{B}-m_{A}$ & $2.24 \pm 0.05$ & & $2.36 \pm 0.05$ & & $2.18 \pm 0.05$ & & $2.37 \pm 0.05$ & $2.36 \pm 0.05$ \\
\hline
\end{tabular}

*Observation date: 2007 October 15

** Observation date: 2007 October 31.

†Observation date: 2006 September 23.

††Observation date: 2006 November 17. 
Table 5. SDSS J1650+4251 Component Positions.

\begin{tabular}{lcccc}
\hline \hline \multirow{2}{*}{ COMPONENT } & \multicolumn{2}{c}{ WIYN* $^{*}$} & \multicolumn{2}{c}{ NOT } \\
& $\Delta$ R.A. & $\Delta$ Decl. & $\Delta$ R.A. & $\Delta$ Decl. \\
& & & & \\
\hline Image A & $\equiv 0$ & $\equiv 0$ & $\equiv 0$ & $\equiv 0$ \\
Image B & $0.223 \pm 0.002$ & $1.163 \pm 0.001$ & $0.24 \pm 0.02$ & $1.17 \pm 0.02$ \\
Lens galaxy G & $0.017 \pm 0.032$ & $-0.872 \pm 0.026$ & $0.015 \pm 0.001$ & $-0.88 \pm 0.07$ \\
\hline
\end{tabular}

*Relative positions to image A obtained by Morgan et al. (2003) with the WIYN $3.5 \mathrm{~m}$ telescope at the Kitt Peak National Observatory.

Table 6. HE 0435-1223 R-band Disk Size and Power-law Index Estimations for $\alpha<0.15$

\begin{tabular}{rrrcc}
\hline \hline & \multicolumn{2}{c}{ Linear Prior } & \multicolumn{2}{c}{ Logarithmic Prior } \\
\multicolumn{1}{c}{$\alpha$} & $r_{s}^{R}$ (light days) & $p$ & $r_{s}^{R}$ (light days $)$ & $p$ \\
& & & & \\
\hline 0.01 & $9 \pm 2$ & $1.3 \pm 0.3$ & $9 \pm 2$ & $\gtrsim 2$ \\
0.05 & $13 \pm 4$ & $1.3 \pm 0.3$ & $7 \pm 6$ & $1.7 \pm 0.3$ \\
0.1 & $7 \pm 6$ & $1.3 \pm 0.3$ & $7 \pm 4$ & $1.3 \pm 0.3$ \\
0.15 & $11 \pm 6$ & $1.7 \pm 0.3$ & $9 \pm 4$ & $1.7 \pm 0.3$ \\
\hline
\end{tabular}

